\title{
Norois
}

Environnement, aménagement, société

\section{Mémoire de la catastrophe volcanique de Pompéi, pratiques touristiques et menace du Vésuve dans les photographies du XIX ${ }^{\mathrm{e}}$ siècle}

Memory of volcanic disaster of Pompeii, tourism and Vesuvius threat in photographs of the 19th century

\section{Delphine Acolat}

\section{OpenEdition}

\section{Journals}

Édition électronique

URL : https://journals.openedition.org/norois/9186

DOI : $10.4000 /$ norois.9186

ISSN : $1760-8546$

Éditeur

Presses universitaires de Rennes

Édition imprimée

Date de publication : 22 novembre 2019

Pagination : 81-99

ISBN : 978-2-7535-7875-3

ISSN : 0029-182X

\section{Référence électronique}

Delphine Acolat, « Mémoire de la catastrophe volcanique de Pompéi, pratiques touristiques et menace du Vésuve dans les photographies du xixe siècle », Norois [En ligne], 251 | 2019, mis en ligne le 02 janvier 2022, consulté le 14 janvier 2022. URL : http://journals.openedition.org/norois/9186 ; DOI https://doi.org/10.4000/norois.9186 


Presses
Universitaires
de Rennes
www.pur-editions.fr
Revue en ligne : http://norois.revues.org

\title{
Mémoire de la catastrophe volcanique de Pompéi, pratiques touristiques et menace du Vésuve dans les photographies du XIX ${ }^{\mathrm{e}}$ siècle
}

\author{
Memory of Volcanic Disaster of Pompeii, Tourism and Vesuvius Threat \\ in Photographs of the $19^{\text {th }}$ Century
}

\author{
Delphine Acolat
}

EA 1161 Centre François-Viète, Université de Brest, 20 rue Duquesne, 29200 Brest (delphine.acolat@univ-brest.fr)

Résumé : L'apparition de la photographie a-t-elle modifié la perception de l'éruption de 79 en tant que catastrophe historique majeure, et du Vésuve en tant que volcan dangereux et encore très actif au XIX siècle? Alors que les guides et récits de l'époque ne négligent pas l'importance du risque volcanique présent, les premières photographies montrent surtout le pittoresque de l'ascension du volcan et sont le relais de l'expression tragique de la terrible mort du passé avec les moulages en plâtre des corps visibles lors la visite émouvante du site de Pompéi.

\begin{abstract}
Did the appearance of photography modify the perception of 79 eruption as major historical disaster, of Vesuvius as dangerous and still very active volcano in the 19th century? Guides and travel's books don't neglect contemporary risk of volcano, but first photographs mostly show quaint ascent of the volcano and relay tragic expression of the terrible death in the past with plaster casts of bodies seen during the stirring visit of Pompeii.
\end{abstract}

Mots clés : risque, archéologie des paysages, volcanisme, tourisme, catastrophe naturelle, imaginaire géographique, Vésuve, Pompéi, Italie

Keyword: risk, landscape archaeology, volcanism, tourism, natural disaster, geographical imagination, Vesuvius, Pompeii, Italy

« Les siècles futurs pourront-ils croire, quand les moissons repousseront et qu'enfin reverdiront ces déserts, que

des villes et des populations sont englouties sous leurs pieds et que les campagnes de leurs ancêtres ont disparu dans la mer incendiée?"

Stace, Silves, IV, 4, 81-83, à propos de l'éruption de 79
Pendant la deuxième moitié de $\mathrm{XIX}^{\mathrm{e}}$ siècle où la photographie se développe (Hannary, 2008), la silhouette du Vésuve se dresse au-dessus des ruines de Pompéi, symbolisant un risque permanent et connu. Pourtant, le volcan est devenu un sujet de curiosité et l'objet d'ascensions et d'aménagements, dont témoignent les photographies, ainsi 
que les guides touristiques. En ces années où les éruptions fréquentes ${ }^{1}$ sont un rappel des risques, la photographie reflète la tentative de rendre l'instant présent de l'éruption. Le Vésuve rappelle aussi à chacun, dans une vision quelque peu romantique de la catastrophe, la disparition brutale d'une cité symbole d'une brillante civilisation, que l'on visite non sans curiosité morbide, ce dont témoigne le succès touristique des fameux corps moulés des Pompéiens aux attitudes figées dans le plâtre par Giuseppe Fiorelli en 1863. À l'ère nouvelle de la diffusion majeure de ces photographies, la conscience du risque d'une grande catastrophe potentielle peutelle être désormais transcrite par l'image du résultat d'une fouille archéologique? Cet article va tenter d'y répondre.

À travers les fonds de photographes professionnels locaux ${ }^{2}$ (Fanelli, Mazza, 2013; Alinari, 2004; Osanna, 2015) comme Giorgio Sommer ${ }^{3}$, Robert Rive $^{4}$, Alphonse Bernoud ${ }^{5}$ ou Michele Amodio ${ }^{6}$, ou d'amateurs, nous tenterons de voir si la mémoire de la catastrophe majeure de 79 a été réactualisée par la photographie naissante, et quel rôle touristique important celle-ci a donné au Vésuve, très actif en cette fin de XIX ${ }^{\mathrm{e}}$ siècle.

1. Le 6 février 1850; le $1^{\text {er }}$ mai 1855, éruption effusive, qui touche les villages de Massa et San Sebastiano; le 28 mai 1858; le 8 décembre 1861, forte éruption effusive et explosive, qui dure tout le mois et cause plusieurs morts; le 15 novembre 1868; le 24 avril 1872, éruption qui touche à nouveau Massa et San Sebastiano : la lave envahit l'Atrio del Cavallo, coupant la retraite à vingt spectateurs imprudents qui périssent; le 4 avril 1906, grande éruption effusive puis très explosive. Le cratère a désormais 800 mètres de diamètre, et c'est un point important car il suscite l'intérêt des photographes.

2. Ces fonds sont consultables assez facilement pour nombre de vues, soit dans les collections des bibliothèques (INHA, BNF, Musée Alinari, etc.), soit sur le marché de l'art, en ligne.

3. G. Sommer vit de 1834 à 1914. Il s'associe à E. Behles à Naples jusqu'en 1872, et est actif dans toute l'Italie de 1857 à sa mort. Voir en ligne, la présentation d'une partie de ses travaux, par G. Fanelli : [http://www. historyphotography.org/doc/Addenda_Sommer_FANELLI.pdf].

4. Robert(o) Rive est actif à Naples et dans le reste de l'Italie, de 1860 à la fin des années 1890. G. Fanelli a entrepris d'établir un catalogue par format pour R. Rive, qui est édité en ligne, avec des addenda, en 2015, 2016, 2017. En ligne, [http://www.historyphotography.org/fotografi_2.html].

5. Alphonse Bernoud est un pionnier de la photographie française, actif essentiellement en Italie de 1840 à 1873. Son studio fut repris par le photographe Achille Mauri, de Naples, qui prit sa succession.

6. Michele Amodio, bien qu'italien, installé à Naples, veut toucher un large public de touristes, et de ce fait, il légende ses photographies en français, sauf après 1890. Il n’a pas publié de catalogue. Il faut donc le recomposer pour ses années d'activité, entre 1860 et 1880 environ. Voir en ligne le récent travail de G. Fanelli : [http://www.historyphotography. org./fotografi/].

\section{LE VÉSUVE FUMANT EN TOILE DE FOND SUR LES PHOTOGRAPHIES}

Jusqu'à l'apparition de la photographie sur les sites de Pompéi et Herculanum vers 1846, dans les peintures et gravures, le Vésuve est présenté en toile de fond de nombreux sujets napolitains et pompéiens. Des gouaches vendues par des commerçants de livres immortalisent de façon invariable les lieux topiques de Pompéi, avec un certain pittoresque : le temple d'Isis, le forum, la « caserne des gladiateurs », avec des gardiens, des femmes élégantes, des étrangers et des chiens (Osanna et al., 2015, p. 100), et l'inévitable Vésuve derrière, rappelant le mode opératoire de la catastrophe de 79. À partir de l'apparition de la photographie calotypique vers $1850^{7}$, dans un premier temps, les photographes amateurs et professionnels ne retouchent le ciel à l'encre sur le négatif papier que pour en faire un fond uni en contraste avec les vestiges pompéiens, où le Vésuve semble rarement nécessaire au sens qu'on veut donner l'image $^{8}$ (figure 1). Les toutes premières photographies montrent par exemple un Vésuve dépassant à peine des murs et colonnes, sans fumée ${ }^{9}$, ou ne montrent pas du tout le vol$\mathrm{can}^{10}$. Puis, très vite, les photographies professionnelles des studios napolitains reprennent les stéréotypes de la peinture ${ }^{11}$, le cadrage par le sud est préféré pour placer le Vésuve ${ }^{12}$ dans l'axe central, et la retouche sur négatif est généreusement utilisée pour accentuer ou rajouter le panache dès que

7. Jusque-là, les premières et rares photographies de Pompéi, en daguerréotypes, c'est-à-dire en positif sans retouche possible sur la plaque de cuivre recouverte d'argent, ne permettent pas d'avoir assez de contraste dans le ciel pour montrer un éventuel panache de fumée. En 1846, Calvert Richard Jones prend par exemple la maison de Pansa et seule la forme lointaine du Vésuve apparaît, très indistinctement, derrière les murs et colonnes.

8. Voir aussi la figure avec la vue de l'intersection de la rue de Mercure, par P. Jeuffrain

9. Tel est le cas de John Shaw Smith, en 1851, qui prend la maison de Pansa sans mettre le Vésuve en valeur puisque celui-ci émerge à peine des colonnes de l'atrium. Même chose chez Alfred Nicolas Normand, en 1851, ou Paul Jeuffrain en 1852. Par contre, Richard Calvert Jones prend, dès 1846, la maison de Salluste avec un axe de symétrie généré par le Vésuve au fond, dont la fumée, à peine visible, va être accentuée dans un exemplaire aquarellé (Collection Hans P. Kraus, New York). Voir aussi Rive $\mathrm{n}^{\circ} 148$ : le forum; $\mathrm{n}^{\circ} 400$ : l'amphithéâtre.

10. Rive $\mathrm{n}^{\circ} 113$ et 117 .

11. Voir les vues peintes de Théodore Duclère, vers 1860, d'Enrico Gaeta, vers 1873, d'Antonio Laezza, vers 1880.

12. Il s'agit en l'occurrence du Gran Cono (Grand Cône), à la forme caractéristique, émergeant au-dessus des vestiges de la Somma, à peine visibles depuis le site de Pompéi. Pour une étude complète de la morphologie du Vésuve et de l'histoire de ses représentations iconographiques, voir le mémoire de master 1 de Julie Morin, soutenu à l'université Paris I en géographie, en 2005. 
possible. Il est significatif de constater qu'il y a eu une évolution dans la représentation du Vésuve et de son rapport au site avec l'apparition de retouches sur négatif à la fin des années 1860 : les premières photographies de G. Sommer, encore associé à E. Behles $^{13}$ (série 301 à 360 au catalogue, en stéréoscopies, comme ici le $\mathrm{n}^{\circ} 343$ ) ne présentent pas encore le panache sur le volcan, alors que par la suite, dans des photographies qui vont rester longtemps au catalogue, Sommer l'amplifie en retouchant le négatif (figures $2 \mathrm{a}$ et $\mathrm{b}$ ).

Parmi les vues canoniques ${ }^{14}$ de Pompéi, celle de l'amphithéâtre permet un cadrage qui dégage le Vésuve en toile de fond, dont le bas des pentes correspond à la largeur de la vue. Dans le premier cliché, figure 2a, (stéréoscopique, n 343), le Vésuve se confond avec le ciel (très noir sur le négatif) et est estompé au loin. Le cliché figure $2 \mathrm{~b}$ (n 1218), au format mezzana (moyen), postérieur, est plus travaillé : grain plus fin, contrastes chromatiques plus profonds, cadrage qui permet de montrer un Vésuve plus net, dans le grand axe de l'amphithéâtre. Il s'agit de montrer non seulement la menace permanente du volcan mais aussi de rappeler les événements de

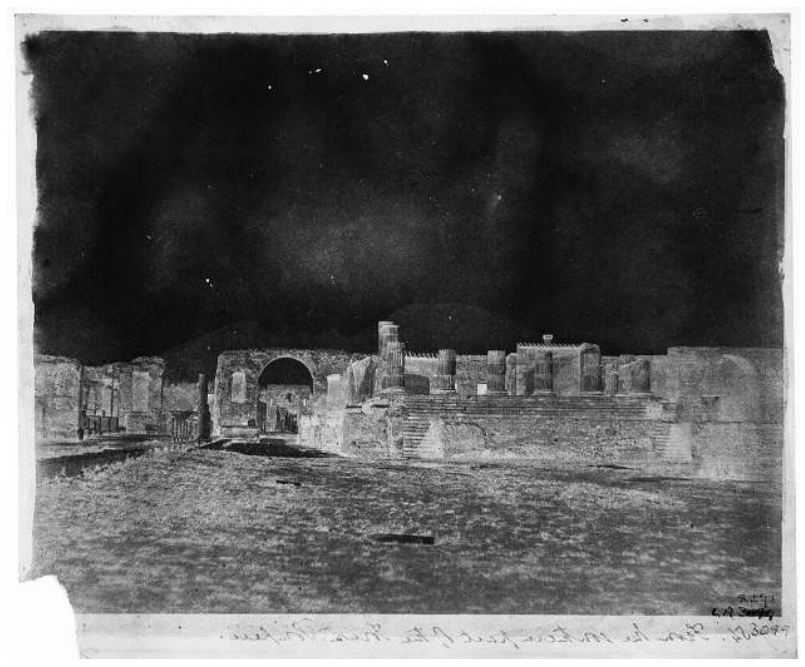

Figure 1 : C.R. Jones, $\mathrm{n}^{\circ}$ 54, négatif : le forum vers le nord avec le Vésuve, peu contrasté par rapport au ciel.

C.R. Jones, $n^{\circ} 54$, forum with Vesuvius.

13. Leur association dure jusqu'en 1874. Outre la vue de l'amphithéâtre que nous présentons, on peut citer celle du forum n 302 en comparaison avec le $\mathrm{n}^{\circ} 1202$ (format moyen).

14. Le Vésuve figure systématiquement derrière les maisons qui ont une orientation nord-sud, les panoramas d'une partie du site, le théâtre et la « caserne des gladiateurs ", les rues, le forum, seulement animé par les ombres de la colonnade. Quand Sommer montre les nouvelles zones fouillées, il les cadre aussi avec le Vésuve (n 10800 : «Scavi nuovi »).
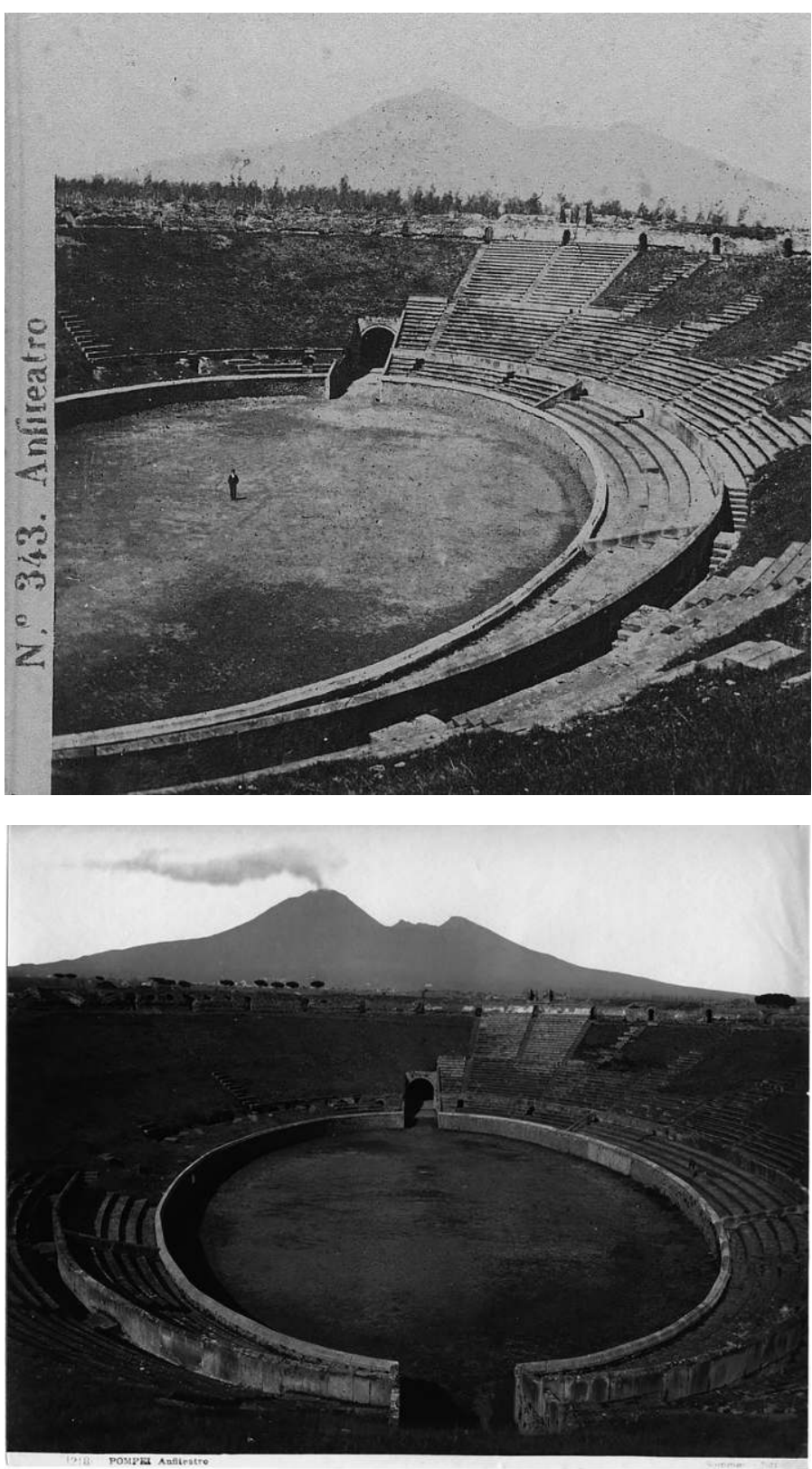

Figure 2 : G. Sommer, (a) no 343 et (b) no 1218 : Pompéi, vue de l'amphithéâtre, avec le Vésuve. with Vesuvius.

G. Sommer, (a) $n^{\circ} 343$ and (b) $n^{\circ} 1218$. Pompeii, amphitheater

79 ayant généré l'état des vestiges au premier plan, volontairement montrés vides de toute présence humaine ( $\left.\mathrm{n}^{\circ} 1218\right)$, puisque rappelant la mort de tous les habitants ${ }^{15}$, ou seulement avec un visiteur, dérisoire face à la masse de l'édifice de spectacle $\left(n^{\circ} 343\right)$. Le contraste est volontairement mis entre

15. G. Sommer, vue de l'amphithéâtre, no 1218 , reste longtemps au catalogue. R. Rive, A. Bernoud, M. Amodio choisissent les mêmes angles et reprennent cet aspect désert. 
le vide du site mis au jour et la capacité de l'amphithéâtre à contenir des milliers de spectateurs.

Une autre vue fréquente est une intersection de la strada de Sallustio avec le Vésuve ${ }^{16}$. Sur la vue de Rive $n^{\circ} 429$ du croisement des rues désertes (figure 3a), le panache est nettement accentué, et le volcan est dans l'axe de la perspective. Cette vue, comparée à celle de Paul Jeuffrain, nettement antérieure, qui avait noirci le négatif sur le ciel de façon uniforme et effacé le volcan (figure 3b), montre la volonté du photographe de mettre en valeur la menace du volcan. Ce même angle de vue vers le nord prévaut dans les clichés des plus célèbres maisons ${ }^{17}$, notamment celle du Faune, qui est un des lieux les plus visités de la ville (figure 4) : la présentation axiale de l'atrium, avec le Vésuve derrière le péristyle, est canonique ${ }^{18}$.

Enfin la vue du forum avec le Vésuve est tellement représentative ${ }^{19}$ qu'elle est reproduite sur certaines couvertures des albums vendus par les photographes (figure 5a); la place publique, lieu communautaire par excellence, est vide de toute vie, sous le Vésuve fumant retouché, évocateur de la destruction, ce qui fait éventuellement contraste avec les dessins montrant la catastrophe et reconstituant l'affolement de la population pendant l'éruption, qui continuent à avoir du succès, et d'ailleurs placés parfois en frontispice des albums de photographies ${ }^{20}$ (figure 5b).

Quant à la ville de Naples, elle est montrée avec le Vésuve éventuellement fumant, de préférence depuis la colline du Vomero ${ }^{21}$ (figure 6), mais la menace semble lointaine, considérée avec une certaine bonhomie. On parle dans les guides du «Vésuve fumant sa pipe » et le volcan ne semble pas mettre en péril la vie napolitaine, volontiers montrée au premier plan, par exemple avec le port

16. Sommer $n^{\circ} 1259$ et $n^{0} 5321$.

17. Autres exemples : Sommer n 84, maison d'Olconius; Bernoud, maison de Pansa.

18. D'autres maisons permettent ce type de cadrage avec le Vésuve menaçant derrière, rappel de la grande éruption, notamment la maison de Pansa, érigée en modèle dans tous les guides touristiques, et étape inévitable d'une visite.

19. Elle figure dans tous les catalogues des photographes napolitains. Entre autres, Sommer, $\mathrm{n}^{\circ}$ 1201, 1202, 1203.

20. Par exemple, album des studios Mauri, héritiers d'A. Bernoud, vers 1880

21. Vues prises depuis la Villa Comunale, la via Marina, la piazza Municipio, Santa Lucia, le Pausillipe ou la colline du Vomero avec la Chartreuse de San Martino, tournées vers le sud-est : par exemple, G. Sommer, $n^{\circ} 1167$ (vue du Vésuve depuis la colline du Vomero). Un pin parasol sert souvent de $l^{\text {er }}$ plan dans des photos très construites, où il y a un contraste recherché entre le panache blanc du Vésuve et le pin se détachant en noir sur un ciel sans nuages (le panache et le ciel uni sont souvent retouchés sur négatif). et les navires (figure 7). Dans les albums intitulés Naples et ses environs, le contraste est ainsi volontairement mis entre la cité morte de Pompéi et la ville de Naples, pleine de vie.

Le voyageur s'attend donc à admirer plus qu'à craindre le Vésuve dans cette seconde moitié du siècle. En 1871, dans sa description de la baie de Naples, l'abbé C. Chevalier fait une remarque qui peut sembler surprenante aujourd'hui, sur le calme du volcan : il cherche, depuis sa fenêtre à Naples, « à y surprendre du regard quelque reste d'activité; mais le volcan s'enveloppait dans une inertie déses-
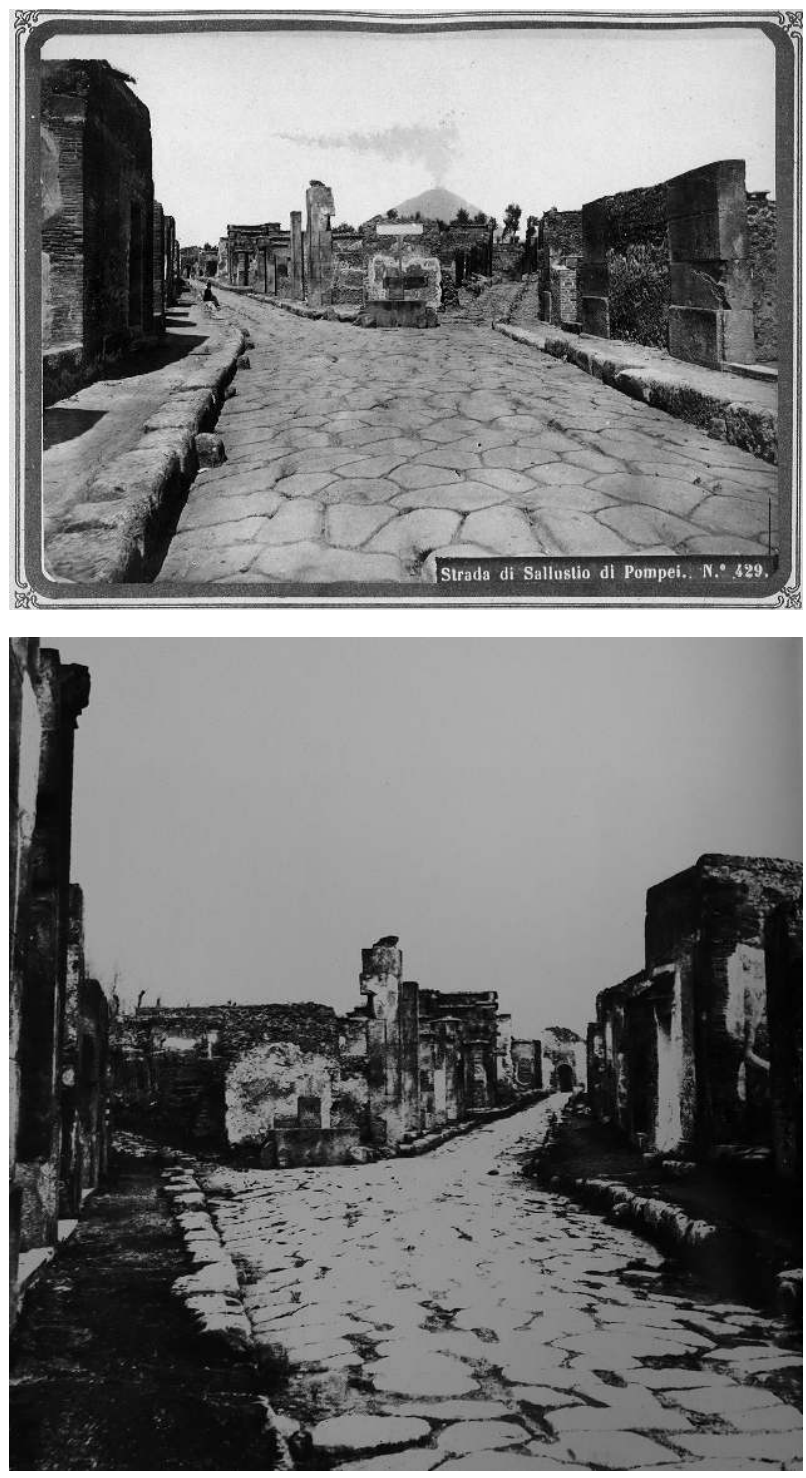

Figure 3 : (a) R. Rive, no 429 : Pompéi, vue des rues avec le Vésuve, vers 1865-1870; (b) P. Jeuffrain : rues, 1852.

(a) R. Rive, $n^{\circ} 429$. Pompeii, streets with Vesuvius, 1865 1870; (b) P. Jeuffrain :streets, 1852. 


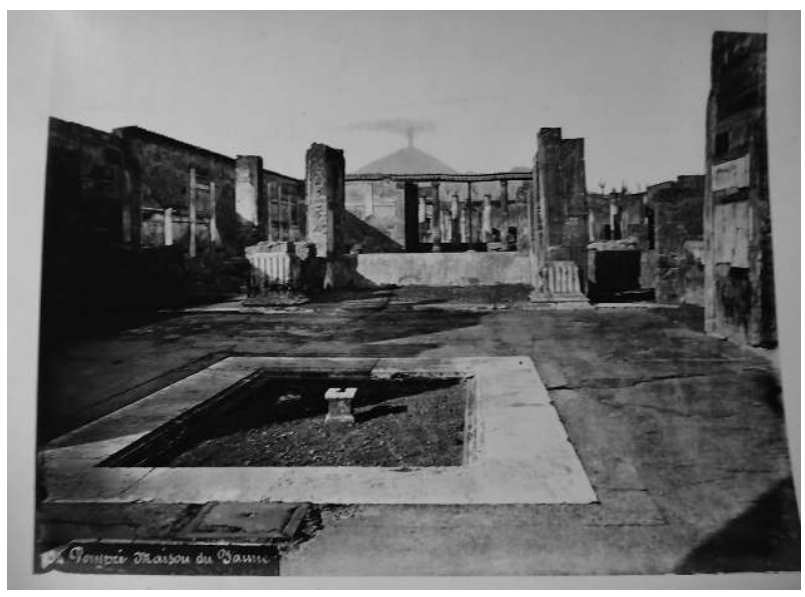

Figure 4 : Bernoud, nº 34 : Pompéi, Maison du Faune avec le Vésuve

Bernoud, $n^{\circ}$ 34: Pompeii, House of the Faun with Vesuvius
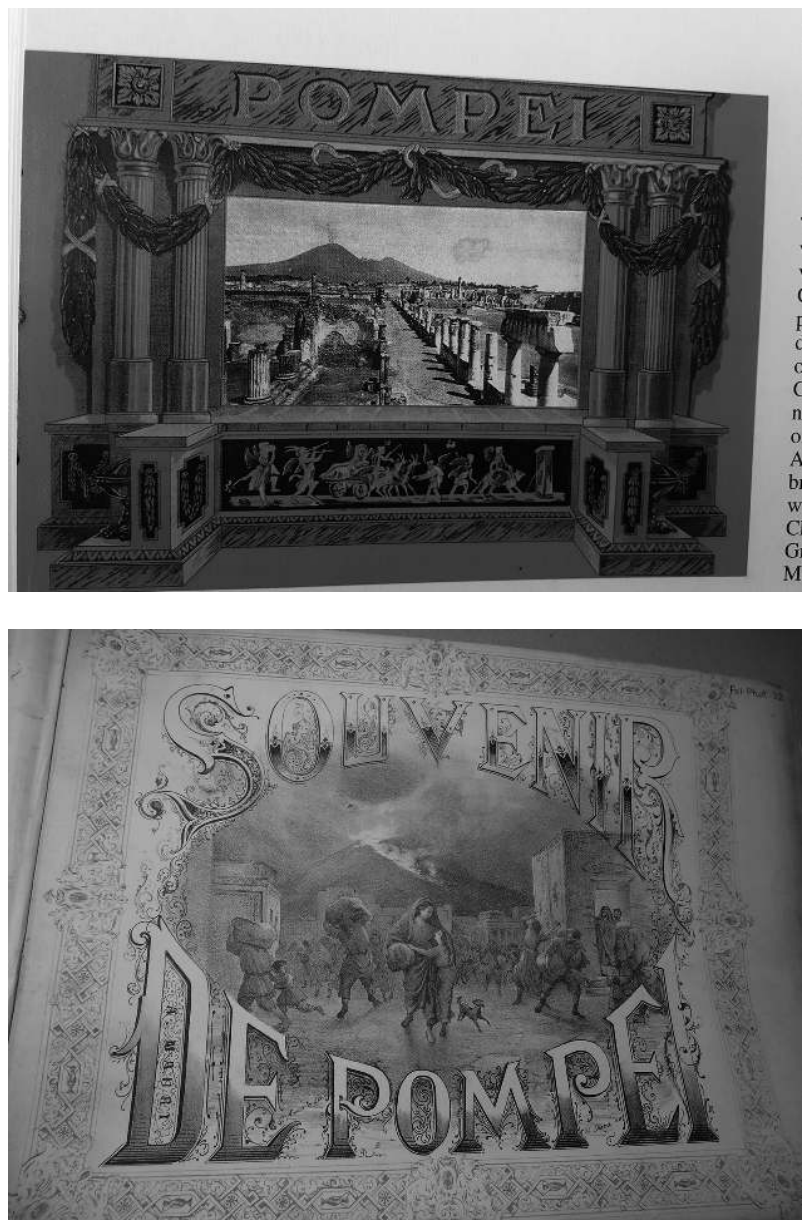

Figure 5 : (a) Album sur Pompéi : couverture, avec le forum et le Vésuve fumant, vers 1880-1900; (b) Frontispice d'un album de Mauri : dessin de reconstitution de l'éruption de 79, vers 1880. (a) Pompeii album: front cover with forum and smoking Vesuvius, about 1880-1900; (b) Frontispiece of Mauri's album: drawing of $79 A D$ eruption, about 1880.

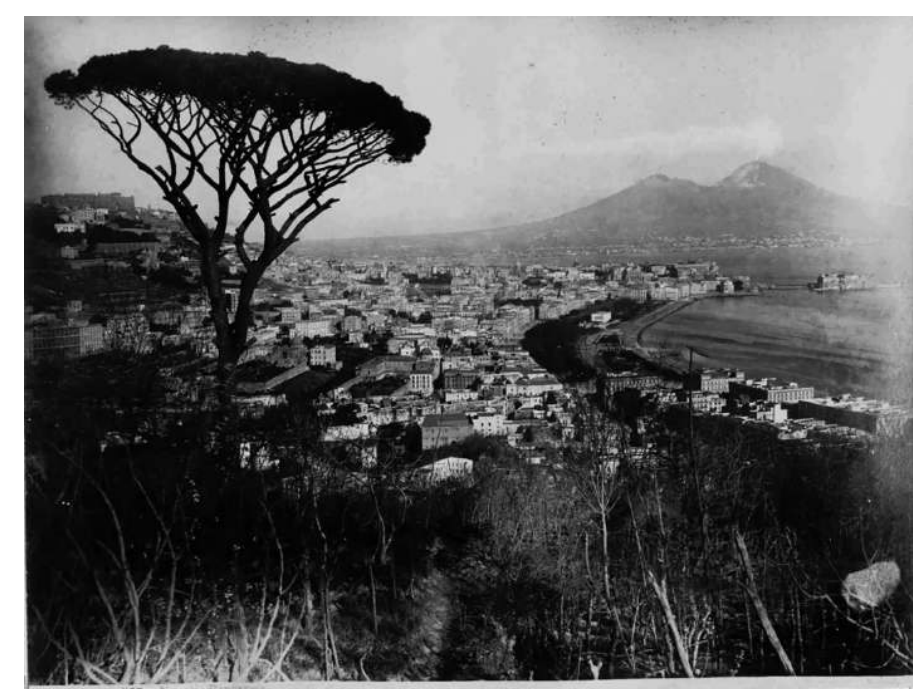

Figure 6 : G. Sommer, nº 1167 : Naples et le Vésuve. G. Sommer, $n^{\circ}$ 1167: Naples and Vesuvius.

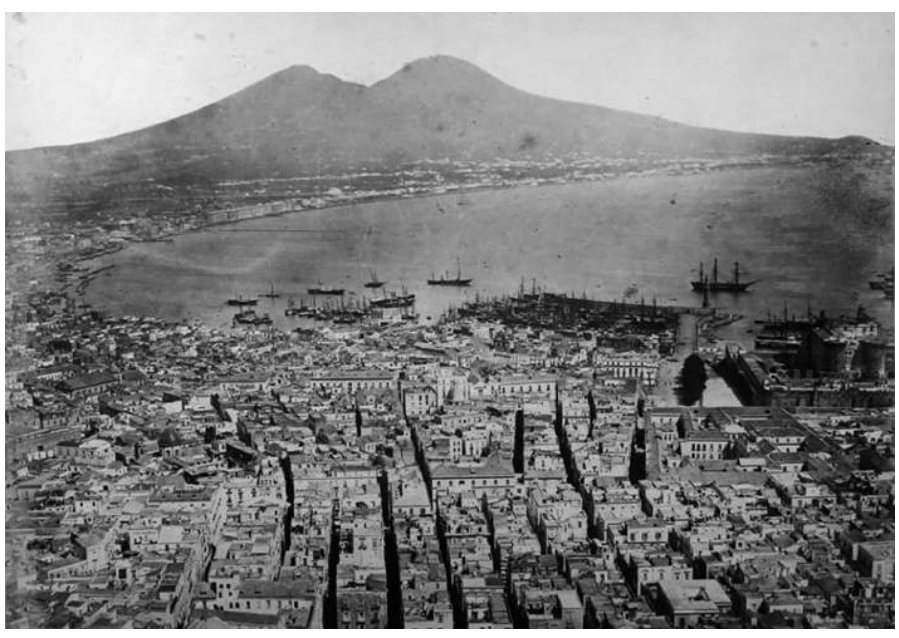

Figure 7 : R. Rive, $\mathrm{n}^{\circ} 1$ : Naples et le Vésuve. Cette photographie est très comparable à celle de Sommer, $\mathrm{n}^{\circ} 1103$.

R. Rive, $n^{\circ} 1$. Naples and Vesuvius.

pérante $[\ldots] »$. Il évoque « cet état actuel de repos et presque de candeur du Vésuve » alors qu'il décrit précisément les fumées, nommées «vapeur » ou « nuage 22 », qui s'en échappent de façon irrégulière. Le Guide Joanne (1855, p. 602), cite «l'insouciance de l'homme sous les menaces de la nature ». C'est seulement une fois au sommet que $\mathrm{C}$. Chevalier semble prendre conscience du danger : «On comprend sans peine qu’à cette faible distance le Vésuve est pour [Naples] une menace et un danger permanents ${ }^{23}$. »

22. Chevalier, 1871, p. 134-135.

23. Chevalier, 1871, p. 197. 


\section{Catastrophe spectacle ET OUBLI DE LA MORT?}

\section{Le volcan examiné et gravi, objet touristique de fascination}

Les visiteurs de la région de Naples ne manquent pas de monter au Vésuve ${ }^{24}$, et si les guides touristiques sont mis à jour sur les éruptions, avant la fin du siècle ils ne contiennent qu'une carte de la région présentant peu d'indications précises sur les coulées antérieures ${ }^{25}$. Lorsque l'activité du Vésuve se résume à des éjections de scories et à des fumerolles, de nombreux voyageurs montent jusqu'au Grand Cône. Les touristes veulent rapporter un souvenir de leur ascension, pour revivre la contemplation émerveillée d'un spectacle naturel qu'ils sont allés approcher de près, en oubliant un peu trop vite parfois le risque réel de mort. Les photos vendues par les studios napolitains répondent parfaitement à cette attente : ce sont des vues soit du volcan seul, soit avec des guides ou des voyageurs au milieu des laves, auxquels les acheteurs peuvent ainsi s'identifier. Il y a même des échantillons de lave qui peuvent accompagner le cliché.

La plupart du temps ${ }^{26}$ depuis la localité de Resina (Herculanum actuelle) où l'on parvient par chemin de fer ou voiture attelée depuis Naples, les touristes montent avec un guide local ${ }^{27}$ au Vésuve par une route qui a été fréquemment interrompue par une coulée puis reconstruite ${ }^{28}$. Les voyageurs montent à pied, à mulet, à cheval, ou en chaise à porteurs

24. Guide Joanne, 1865, p. 375 : « Après avoir vu Naples, le premier but offert à l'impatiente curiosité du voyageur n'est-il pas le Vésuve et Pompéi?"

25. Entre autres exemples, le Guide Joanne, 1853, p. 583. Il rappelle ses précédentes éruptions, les voies de montée vers le cratère, la fertilité de la lave, la forme du cratère. La carte proposée n'est pas très récente, et c'est encore le cas pour l'édition de 1859. Le Guide Baedeker de 1869 présente encore une carte peu précise (p. 89) sans datation des coulées. Mais à la fin du siècle, les différentes éditions vont faire une mise à jour des informations géographiques. Le Guide Baedeker de 1912 présente un bilan des éruptions du XIX ${ }^{\mathrm{e}}$ siècle, cartographié sur une double page ayant pour titre Contorni di Napoli.

26. Il y a deux alternatives à la voie depuis Résina : l'une au départ de Pompéi, d'où l'on monte à cheval en $2 \mathrm{~h} 30$ à proximité du cratère. Le Guide Joanne de 1865 précise que «l'on paie $10 \mathrm{fr}$ pour le guide et le cheval, qui attendent le voyageur pendant l'ascension au sommet ». L'autre, au départ de Torre Annunziata (Guide Murray, p. 183).

27. Le Guide Joanne, en 1865, explique que c'est à Résina qu'on trouve « des guides, des porteurs et des montures », et il donne leurs coûts respectifs (p. 375). Le Guide Murray de 1862 conseille un guide en particulier : "Pasquale Gozzolono est le seul guide à avoir une connaissance scientifique sur la montagne » (p. 183), et il critique les «self-called guides » qui « importunent» le voyageur, et les imposteurs.

28. En 1865, le Guide Joanne, p. 375, précise que depuis Résina il y a jusqu'à l'ermitage « une belle route neuve; les piétons peuvent abréger en coupant les détours de la route». (figure 8) (2 heures jusqu'à l'ermitage ${ }^{29}$, puis 2 heures encore jusqu'au cône, à pied), puis à partir de 1880, avec le funiculaire de la partie sommitale ${ }^{30}$.

À travers les mises en garde des guides contre les «brigands ", on constate que le Vésuve est une manne pour les Napolitains, qui figurent sur certaines photos. La route passe par l'Observatoire vulcanologique (Osservatorio meteorologico Vesuviano) récemment inauguré en 1845, et fréquemment photographié soit comme sujet principal, soit comme arrière-plan (figure 8) pour évoquer l'ascension et l'étape obligée, associée à la dégustation du célèbre vin nommé lacryma Christi ${ }^{31}$ près de l'ermitage San Salvatore. On peut avoir accès à la collection de l'observatoire, pour y admirer des minéraux, mais cette dernière n'est jamais photographiée.

Lors de l'ascension, nombreux sont les touristes qui se prêtent à des expériences à l'instigation de leur guide local. Les photos retranscrivent ces pratiques attestées par les guides touristiques et récits ${ }^{32}$, notamment celle qui consiste à enfoncer un bâton sous la croute de lave refroidie à moins

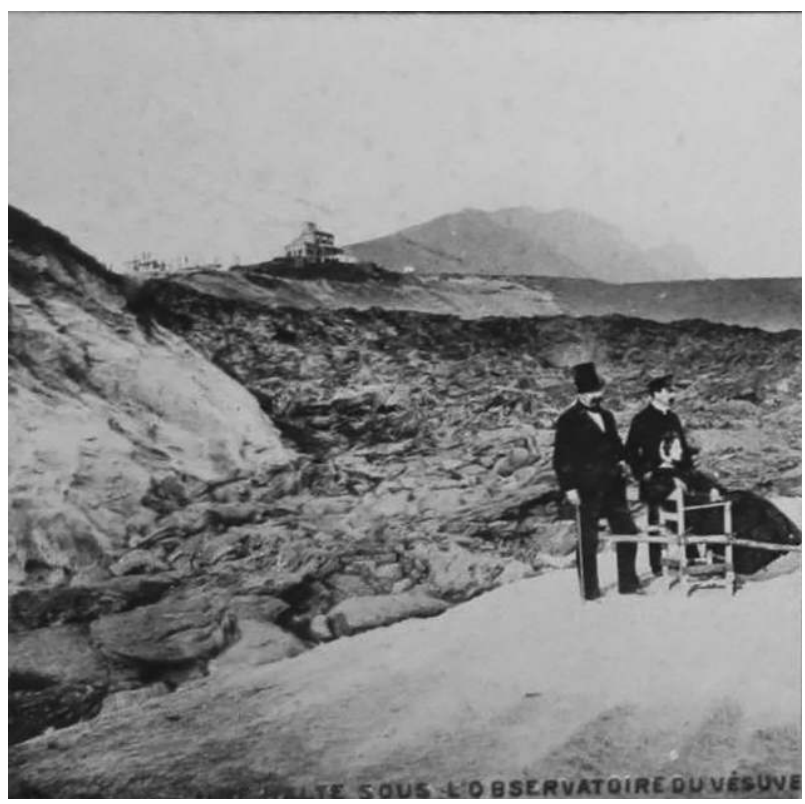

Figure 8 : Anonyme. Route vers le Vésuve avec chaise à porteur. Halte sous l'Observatoire vulcanologique. der Observatory.

Anonymous. Road to Vesuvius with sedan chair. Halt un-

29. Guide Joanne (1865), p. 375

30. Voir texte du Guide Baedeker en annexe.

31. Anonyme amateur, en stéréoscopie : le chemin qui mène à l'Observatoire et des paysans vendeurs de vin

32. Guide Joanne, 1865, p. 378. 
d'un mètre de profondeur et de le voir s'enflammer (figure 9) ou acheter un œuf au pied du cône final " pour le faire cuire dans une fissure du cratère ${ }^{33}$ ".

Les journaux se font l'écho de telles pratiques qui oublient tout risque et présentent l'excursion au Vésuve sous son meilleur jour, à l'exemple de l'Illustrated London News, en 1872. Il faut monter au Vésuve «pendant qu'il travaille ${ }^{34}$ » et éjecte des scories.

Avant l'installation du funiculaire en 1880, l'ascension finale du cône du Vésuve n'est pas chose aisée, les guides touristiques avouent même qu'elle est « très pénible » (Guide Baedeker, 1896) et « extrêmement fatigante » (Guide Joanne, 1865), nécessitant trois quarts d'heure à elle seule. Certains touristes ont recours au portage, comme en atteste la photographie de Sommer des années 1880, très scénographiée (figure 10).

On pose seul ou en groupe. Que ce soit dans les catalogues de photographies vendues par les studios professionnels (Sommer, Amodio, Bernoud, Brogi, Rive) ou dans des albums réalisés de façon amateur ${ }^{35}$, l'accent est donc mis sur le côté pittoresque de l'ascension, les touristes s'approchent dangereusement des bords du cratère principal ou des cônes secondaires pour poser près des fumerolles (figure 11).

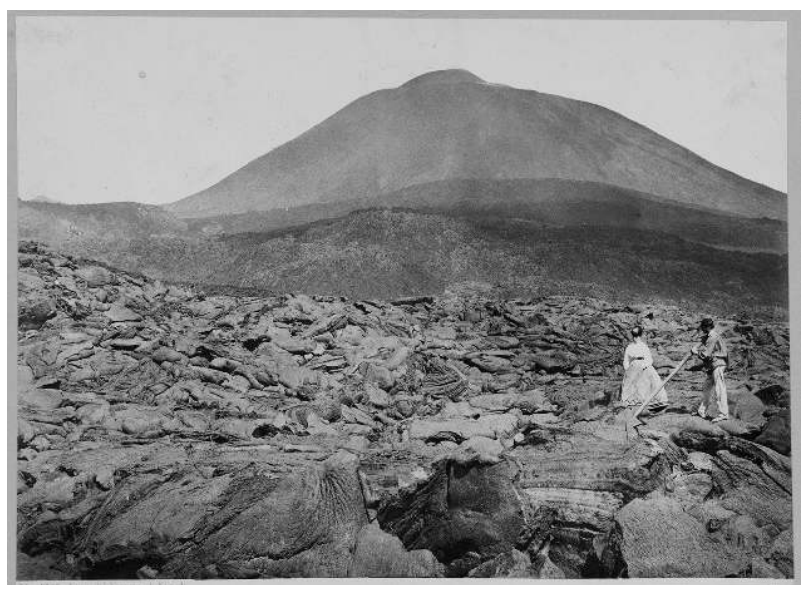

Figure 9 : G. Sommer, no 6138. Le Vésuve avec une touriste et un guide au milieu des laves. Le $\mathrm{n}^{\circ} 6139$ montre la même scène mais avec des positions légèrement différentes. guide in lava.

G. Sommer, $n^{\circ}$ 6138. Vesuvius with one tourist and a

33. Guide Baedeker, 1869, p. 108

34. Guide Baedeker, 1869, p. 113.

35. L'un d'entre eux, que nous avons consulté, malheureusement anonyme, précise la date et l'heure de chaque cliché.

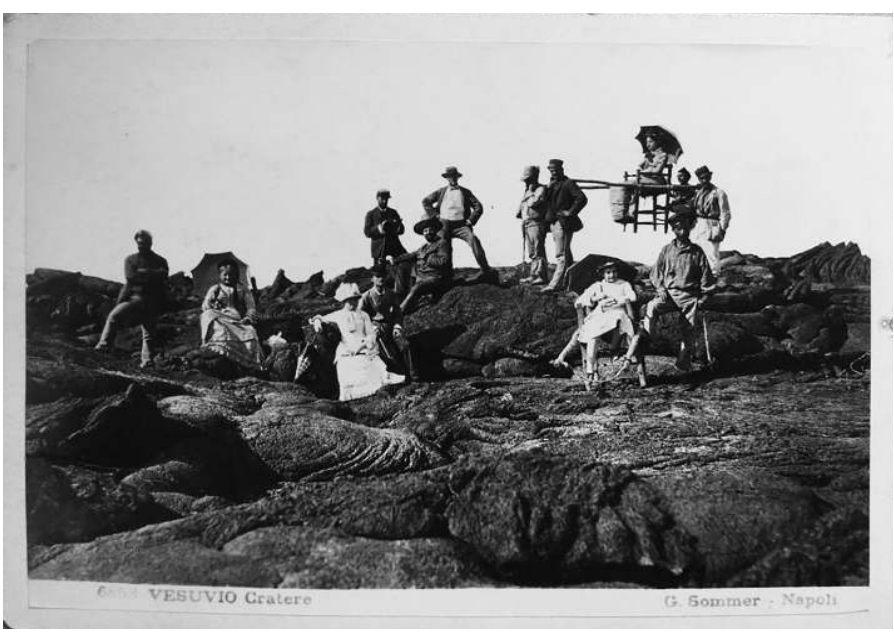

Figure 10 : G. Sommer, $\mathrm{n}^{\circ}$ 6858. Le cratère du Vésuve avec des touristes et des chaises à porteur.

chairs.

G. Sommer, $n^{\circ}$ 6858. Vesuvius crater with tourists and sedan

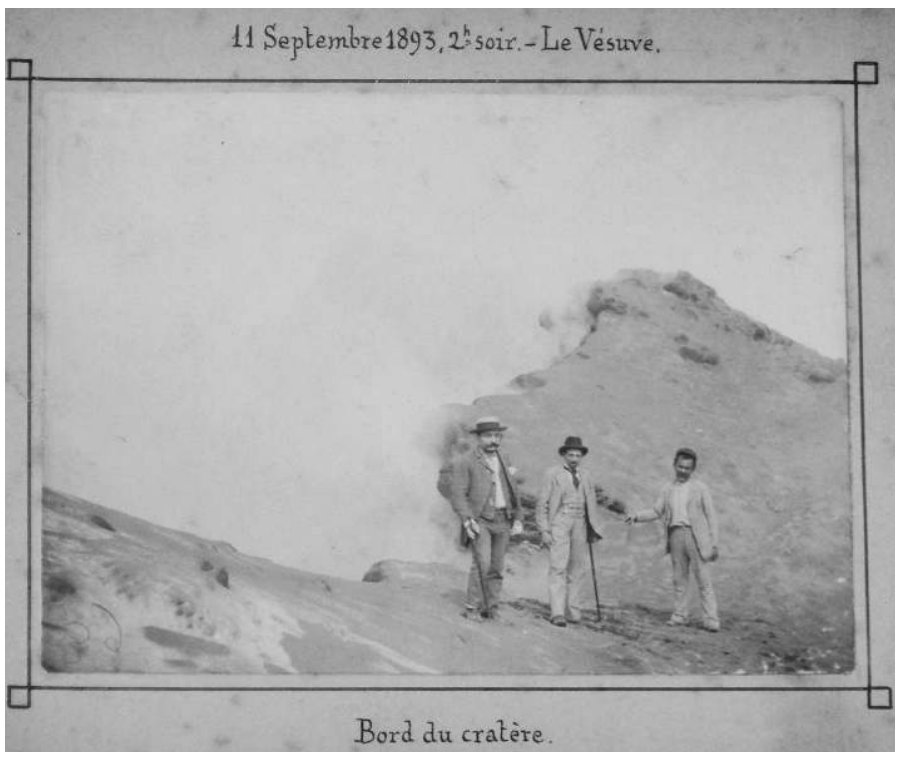

Figure 11 : Album amateur anonyme. « 11 septembre 1893, Le Vésuve, 2h du soir, bord du cratère. »

Anonymous. "1893, september 11th Vesuvius. 2 p.m., crater's rim."

À partir du moment où le funiculaire existe ${ }^{36}$, les photographies servent de témoignage sur la performance technique de l'ouvrage, sur l'amusement et le confort apporté par l'utilisation de ce nouveau moyen motorisé d'ascension qui mène les touristes à 200 mètres sous le sommet. G. Sommer

36. Il est inauguré le 6 juin 1880, fait $830 \mathrm{~m}$ de long et gravit un dénivelé de $390 \mathrm{~m}$ sur la face sud-ouest, sur une pente entre 45 et $63 \%$. Il est ouvert au public le 10 juin 1880. 
et A. Mauri, en particulier, proposent dans leur catalogue plusieurs clichés des wagons nommés Etna et Vésuve ${ }^{37}$, ainsi que des gares supérieures et inférieures, avant qu'elles ne soient détruites par une coulée de la grande éruption de 1906 (figures $12 \mathrm{a}$ et $\mathrm{b}$ ).

Juste après l'inauguration, une chanson napolitaine est même créée pour inciter les gens à ne rien redouter du volcan et à considérer l'ascension
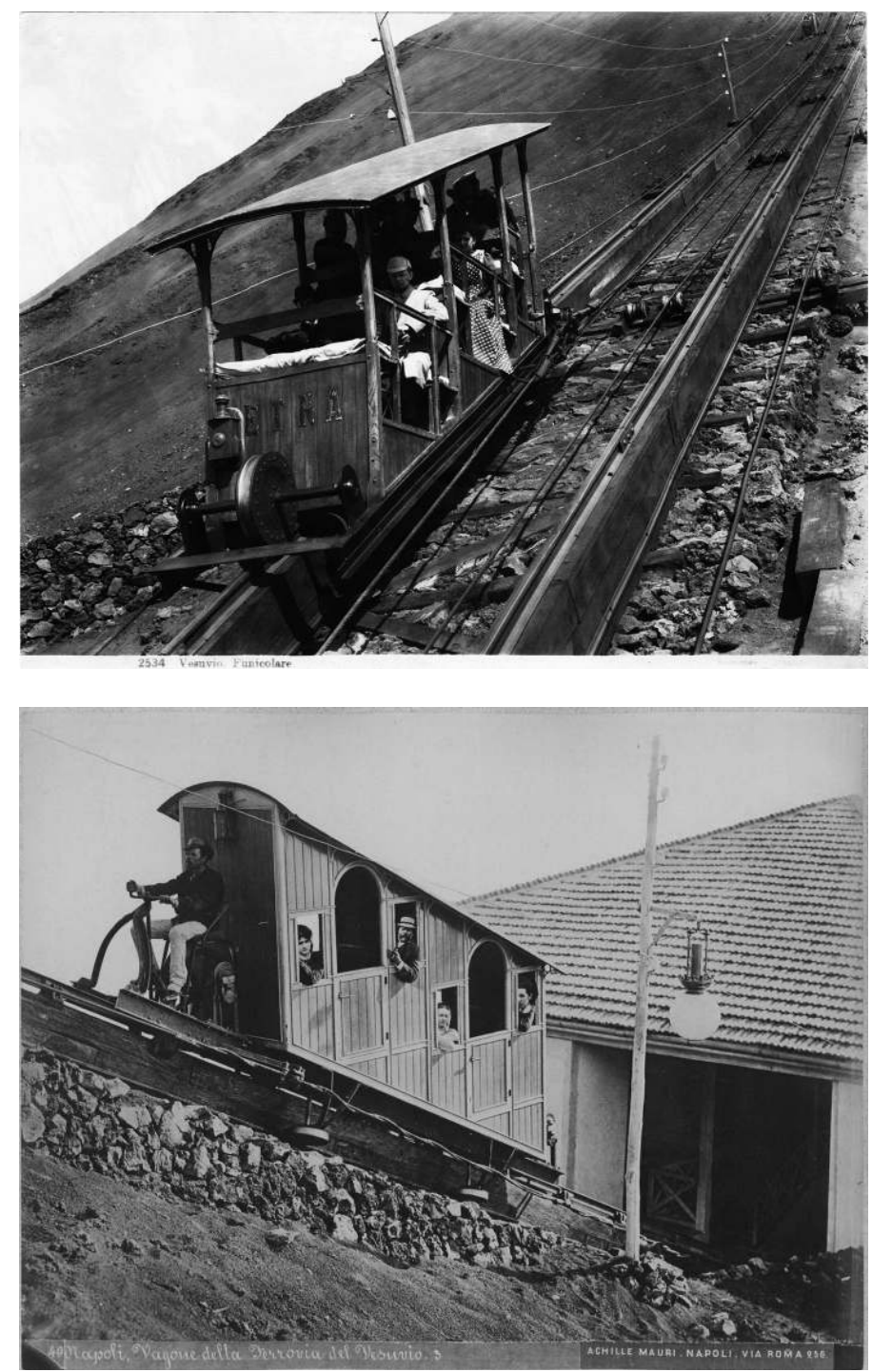

Figure 12 : Le funiculaire. On voit déjà dans ces clichés deux générations de wagons; (a) G. Sommer, no 2534. Le funiculaire en 1880 (première génération de wagon); (b) A. Mauri, no 40. Funiculaire (deuxième génération de wagon). Funicular.

(a) G. Sommer, $n^{\circ} 2534$. Funicular in 1880; (b) A. Mauri, $n^{\circ} 40$.

37. Sommer no 2534 et 6852 . Mauri, no 39 et 40 . comme un jeu et un objet de curiosité renouvelée ${ }^{38}$. Seul le restaurant-buvette qui se trouve au pied de la station inférieure peut évoquer, par son architecture qui se veut à la pompéienne, le rapport avec la catastrophe de 79 et la cité romaine disparue, mais en fait les photographies ne le montrent guère.

\section{Photographies d'éruptions et idée de catastrophe}

Lors des périodes éruptives, les voyageurs manifestent une grande fascination pour le spectacle qualifié de «théâtral » dans les guides ou dans les récits de voyageurs. Ceci dit l'absence de couleurs en photographie ne permet pas une théâtralisation de l'éruption aussi marquée que dans les peintures et aquarelles, où la nuit permet de mettre en valeur l'embrasement des coulées et les éjections. Tout au plus peut-on voir en photographie des fumées blanches. Lors des éruptions, le vocabulaire employé est antinomique : on parle de «belle horreur ", d' « horreur sublime ${ }^{39}$ ", que l'on regarde de loin, en général depuis Naples, ou de près pour certains, plus téméraires, qui osent monter lors des éruptions, négligeant bien souvent le danger. Cette perception «ludique » de l'éruption et la négation du danger est celle que préfèrent transmettre les journaux qui utilisent désormais les photographies des studios napolitains, à l'exemple de L'Illustration du 20 juillet 1895, publiant une gravure tirée des photographies des Edizioni Esposito, montrant les touristes fourmillant autour des coulées fumantes (figure 13).

Lors de l'éruption d'avril 1872, le docteur Guiraud explique avoir dû renoncer à monter jusqu'au cône. Il atteint « la lave dont l'extrémité était dans l'Atrio del Cavallo » mais son texte est sans équivoque : on s'amuse sans penser au danger, on fume, on boit et « cette nuit-là, le Vésuve était un but de promenade

38. Un journaliste, Peppino Turco et un musicien, Luigi Denza composent cette chanson en italien, qui fera par la suite le tour du monde, dont voici le début en traduction française : "Hier soir, Annette, je suis monté. Devine où? Où ce cœur ingrat ne peut plus m'embêter! Là où le feu brûle, mais si tu te sauves, il te laisse tranquille, et ne te poursuit pas, ni ne se lasse de contempler le ciel! Allons, allons, au sommet allons-y! Allons, allons, au sommet allons-y! Funiculi, funiculè, funiculi, funiculaire! Allons au sommet, funiculì, funiculaire! Allons de la terre à la montagne, il n'y a qu'un pas. On voit la France, Procida et l'Espagne, moi je te vois toi! Avec le funiculaire, aussitôt dit aussitôt fait, nous sommes montés au ciel... [...]»

39. Chevalier C., 1871, p. 198. 


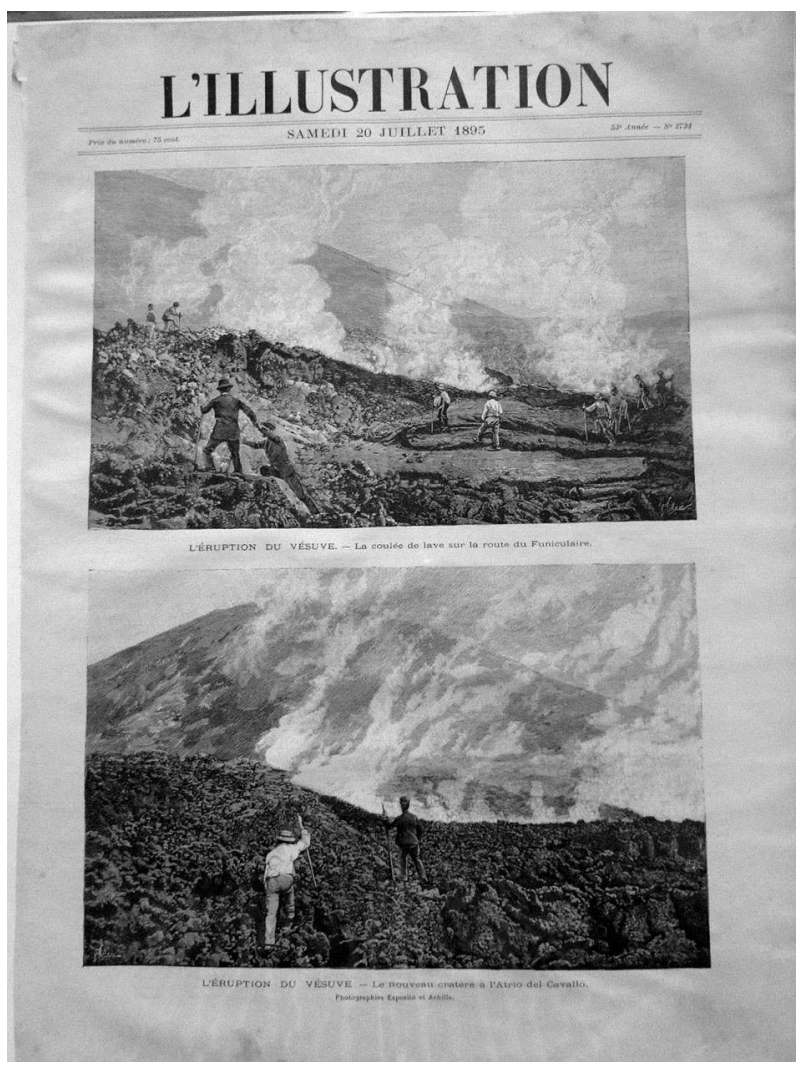

Figure 13 : L'Illustration du 20 juillet 1895 : une éruption et des touristes, d'après photographie d'Esposito Esposito's picture

L'Illustration, 1895, July 20th: eruption and tourists from

où se dirigeait une partie de Naples ${ }^{40} »$. Pourtant, Guiraud raconte qu'il a appris le lendemain que suite à l'ouverture d'un cratère dans l'Atrio del Cavallo, une coulée brutale a enseveli une vingtaine de spectateurs. C'est aussi ce que narre un article du journal L'Illustration de juin $1872^{41}$ joint à une photographie de Bernoud faite depuis Naples qui montre la triple coulée (figure 14), avec un champ lexical fondé sur la peur et le danger (« épouvanté, effrayant, redoutable, menaçant, terrible »). La photographie est ici une garantie de la véracité de l'information sur l'éruption.

Ces photographies d'éruption, rendant enfin compte de la réalité grâce à l'instantanéité, vont faire sensation, notamment parce qu'un séquençage d'éruption a lieu pour la première fois. G. Sommer photographie en effet l'éruption toutes les demiheures. Le succès de ces clichés ne va pas être provisoire : ils figurent encore à son catalogue de

40. Guiraud, 1872, p. 15-16. Voir en Annexe, le texte du Guide Baedeker. 41. L'Illustration, $\mathrm{n}^{\circ} 1524,11$ juin 1872, p. 292-294.

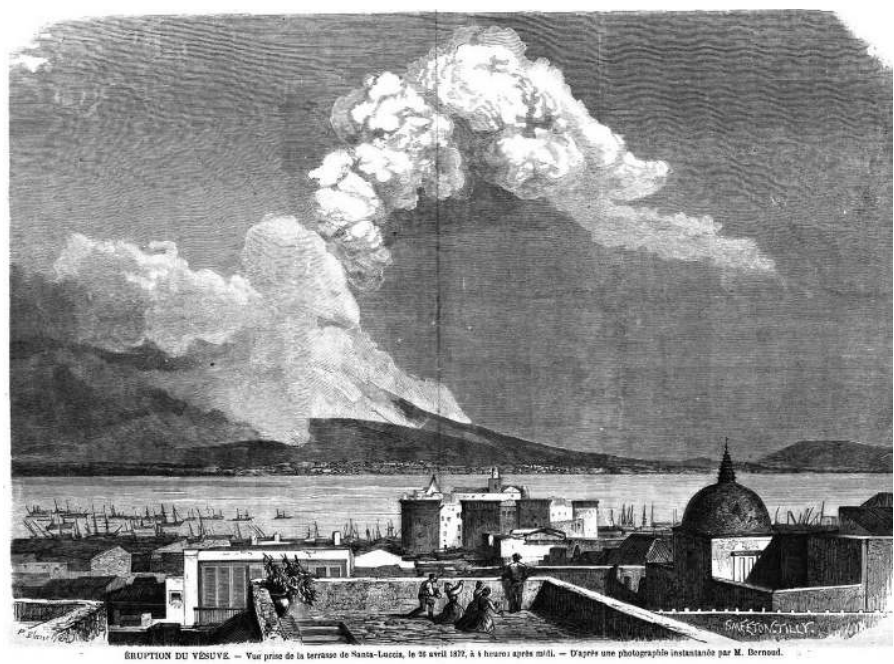

Figure 14 : L'Illustration du 11 juin 1872 : l'éruption de mai d'après photographie de Bernoud noud's picture

L'Illustration, 1872, June 11th: the eruption of May, from Ber-

1912 et sont reproduits dans nombre d'ouvrages de référence sur Pompéi (Gusman, 1902, p. 13). La photographie permet aussi de rendre compte rapidement des destructions, notamment en 1872, dans San Sebastiano et Massa, composant un véritable reportage ${ }^{42}$ où des hommes, figurent, servant d'échelle (figure 15), dont le succès et l'intérêt est prouvé par la publication dans les journaux sous forme de gravures ${ }^{43}$.

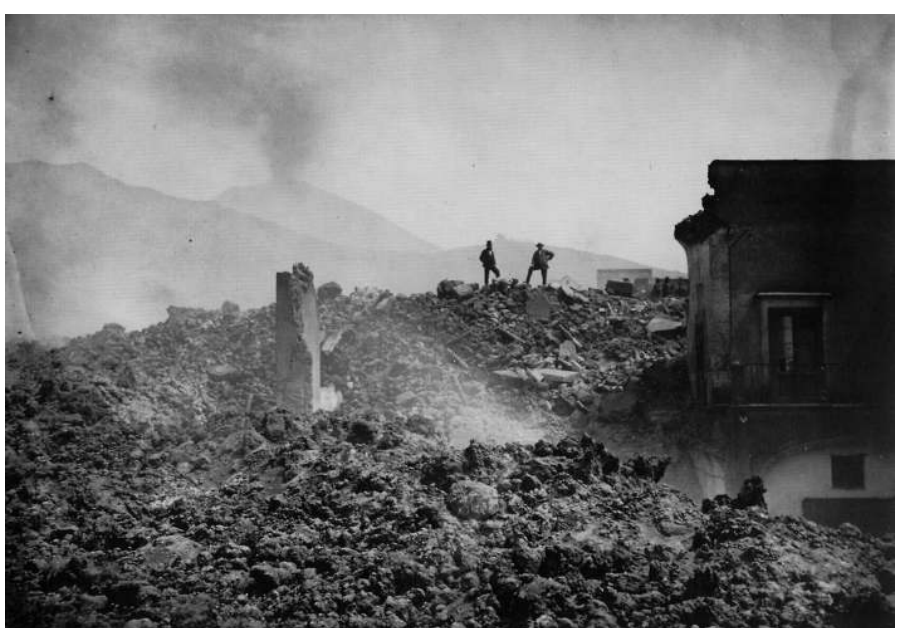

Figure 15 : G. Sommer, destruction par la lave à San Sebastiano, 1872 G. Sommer, lava's destruction in San Sebastiano, 1872

42. Sommer, album Naples et ses environs, BNF. Consultable sur Gallica. 43. Légende : « d'après photographie de G. Sommer, Napoli ». 
Rive et Sommer sont allés sur les lieux alors que les laves sont encore fumantes. Même si, ensuite, il y a eu d'autres éruptions, Sommer propose toujours dans son catalogue ces photos marquantes plusieurs années après ${ }^{44}$, pour montrer la puissance dévastatrice du volcan. Or cette violence éruptive du Vésuve, rarement mortelle au XIX ${ }^{\mathrm{e}}$ siècle, est cependant rappelée comme telle sans cesse sur le site de Pompéi à partir de 1863, notamment à cause des moulages des corps.

\section{« LA NATURE A ÉTÉ MOULÉE SUR LE VIF ENTRE LA MORT ET L'AGONIE » : LES PHOTOGRAPHIES DES MOULAGES DES CORPS DE POMPÉI}

\section{Une mise en scène des moulages par les photographes}

En 1832, en visite à Pompéi, Walter Scott ne cesse de répéter qu'il voit « la Ville de la Mort ». En 1867, E. de Kératry écrit que « c'est à sa mort que Pompéi doit son immortalité », et lors d'une visite, «vous sentirez alors que tout a été plein de vie ${ }^{45}$ ». Entre ces deux dates, une même perception d'une ville figée dans l'instantanéité de la mort, mais les photographies relaient désormais un rapport permanent entre la mort d'autrefois, figée et montrée par des techniques nouvelles en archéologie, et la menace permanente de mort due au Vésuve très actif, confrontée avec une vie nouvelle sur le site archéologique, due au tourisme.

L'idée même du traitement du corps du mort a évolué début XIX ${ }^{\text {e }}$ siècle avec les progrès de la science archéologique. Sur les photographies de Pompéi avant 1863, la mort brutale des habitants de la ville en octobre 79 est, nous l'avons vu, d'abord seulement perçue via l'absence d'êtres vivants, systématiquement recherchée, en contraste avec les lieux publics et l'intense activité qu'ils suggèrent ${ }^{46}$ (figures 1, 2, 3 et 4). Or la technique de fouilles suivie par Giuseppe Fiorelli change les choses de façon révolutionnaire le 5 février $1863^{47}$, même si, dès 1831 , une première mais vaine tentative avait eu lieu pour mouler l'empreinte du corps d'une femme

44. Sommer, catalogue de 1886 (2 clichés : nº 6113 et 6112 ). 45. Les ruines de Pompéi, Paris, 1867, p. 9 et 27.

46. Notamment les panoramas de rues (Sommer $n^{\circ} 1259$ ), le forum (Sommer, n $302, n^{\circ} 1202$, ou Mauri, n 106), ou l'amphithéâtre.

47. Layard, 1864, p. 161-180. trouvé dans la maison du Faune. C'est donc plus de 100 ans après le début des fouilles et la découverte de nombreux squelettes ${ }^{48}$ que l'on va proposer une lecture de l'instant de la mort des Pompéiens avec le moulage en plâtre autour des squelettes, dans l'empreinte des corps dans la cavité laissée par les matières volcaniques de la nuée pyroclastique solidifiée $^{49}$. Le succès photographique des moulages des corps des Pompéiens ajoute au site une interprétation dramatique du moment de la mort en 79 . L'impuissance de l'homme face à une nature violente personnifiée par le Vésuve, symbole de mort, est désormais aussi transmise par l'image, nouvelle et photographique, des moulages des morts de 79 montrant leur agonie poignante ${ }^{50}$. Lecture morbide, lecture subjective (Gardner Coates, 2012), puisque les commentateurs et auteurs vont prêter aux corps une histoire qui est interprétée d'après des postures que l'on lit parfois très mal, et qui, depuis peu, a souvent été battue en brèche (Lazer, 2017) ${ }^{51}$.

Depuis le début des fouilles, on trouve en effet des squelettes à Pompéi, qui ne sont que très rarement photographiés et ne figurent pas dans les catalogues des studios professionnels. La photo de Michele Busco (figure 16) est à cet égard, en 1885, une exception, puisque depuis février 1863, ce sont les moulages des empreintes de corps dans les matières volcaniques solidifiées qui sont devenues l'objet des prises de vues et un grand succès de vente ${ }^{52}$.

48. À ce jour, on a trouvé environ 1150 corps à Pompéi. 103 ont été moulés (Lazer, 2017)

49. En 1856 on réussit à mouler du mobilier. De 1863 à 1872, 6 moulages d'humains seulement sont réalisés: Fiorelli se heurte à des difficultés techniques, des moulages sont en morceaux, les lapilli de la couche sous les corps empêchent de reproduire correctement les formes. Dwyer, 2010. Pucci 2015. Garcia y Garcia 2006.

50. Cela explique sans doute pourquoi une reconstitution de spectacle de gladiateurs à l'amphithéâtre en 1884 par Giulio De Petra a eu relativement peu de succès : la coexistence avec le rappel constant des morts (par les moulages) est troublante. Sommer prend une photographie de l'amphithéâtre où l'on voit des spectateurs sur les gradins qui subsistent, assistant au combat dans l'arène (Collection Alinari, Florence), mais elle ne figure pas dans son catalogue.

51. Les restaurations de 2015 (86 des 103 moulages furent restaurés) et le passage au scanner et rayons X de 30 moulages faits au XIX ${ }^{\text {e }}$ siècle en septembre 2015, complété par le scan de 5 autres moulages en juin 2017, ont permis de réviser en profondeur des théories sur le sexe et des présomptions sur «l'histoire » des morts. Voir the Pompeii Cast Project mené par l'université de Sydney. [http://www.castprojectpompeii.org/] et les publications d'E. Lazer, 2017. Des ossements furent retirés avant le moulage et remplacés par des barres de métal pour renforcer la solidité des moulages.

52. Ce succès de vente ne se dément pas encore aujourd'hui, puisque sur le marché des collectionneurs de photographies anciennes de Pompéi, les photographies des moulages sont deux à trois fois plus chères que les photographies de vestiges strictement archéologiques. 


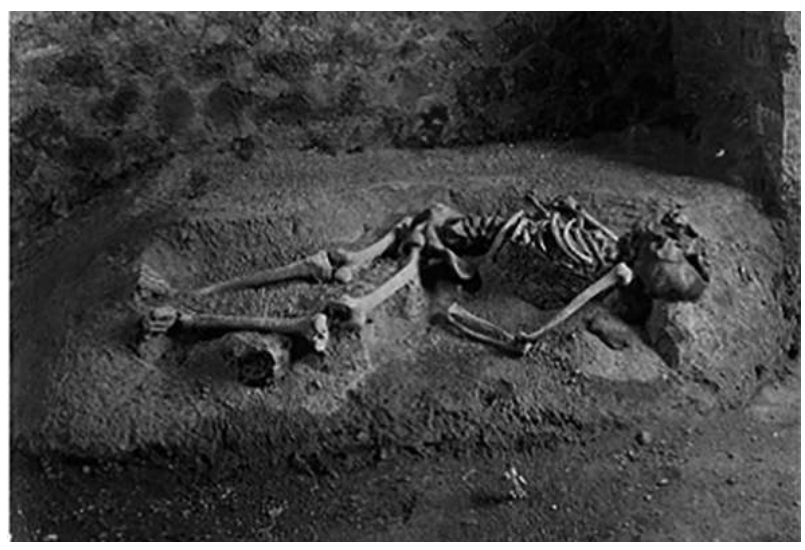

Figure 16 : M. Busco, «Pompei. Scheletro nelle vie dell'abbondanza ", 1885 Napoli, Archivio fotografico della Soprintendenza per i Beni Archeologici di Napoli

1885 .

M. Busco, "Pompei. Scheletro nelle vie dell'abbondanza",

Les photographies de Sommer, Rive, Amodio ou Bernoud sont le media qui révèle au public la violence de la mort lors de l'éruption de 79. Dans les légendes des albums de photographies, on les appelle tantôt «les squelettes », «les morts », les «corps ", mais plus souvent les « empreintes (humaines)» (impronte umane), et peu souvent, « les moulages », ce qui révèle l'aspect technique sans l'idée de la mort, alors qu'on veut insister sur l'aspect novateur de ce qui est de l'archéologie de l'être humain. Luigi Settembrini, homme de lettres et ami de Fiorelli, écrit dans le Giornale di Napoli du 17 février 1863, après les quatre premiers moulages : "Il est impossible de voir les trois figures extraordinaires sans se sentir ému : en particulier la petite fille $[\ldots]$ elle te fend le cœur [...]. Là il ne s'agit pas d'art, il ne s'agit pas d'imitation; [...] c'est la douleur dans la mort qui acquiert de nouveau un corps et une figure [...] Toi, ô mon Fiorelli, tu as découvert la douleur humaine, et quiconque est homme la perçoit aussi ${ }^{53}$. » Or si Fiorelli, avec sa technique ${ }^{54}$, a vraisemblablement voulu d'abord apporter des connaissances scientifiques à l'étude de la catastrophe, en voulant reconstituer et interpréter les causes et modes opératoires de la mort des Pompéiens, il s'avère qu'il se sert aussi des photographies des moulages pour montrer les progrès de ses fouilles au ministère ita-

53. L. Settembrini, Scritti vari di letteratura politic ed arte, I, Napoli, 1879, p. 331-338.

54. Il a moulé 4 corps en 1863, puis son rapport de fouilles montre qu'il y a des échecs répétés jusqu’en 1873. Voir Dwyer, 2010. lien qui le finance ${ }^{55}$. L'énorme impact émotionnel éprouvé par les visiteurs est relayé et amplifié par la photographie : Amodio, Sommer, Bernoud et Rive cherchent à saisir la plus grande expressivité dramatique possible (Zanchetti 2015, p. 366).

L'interprétation des moulages via la photographie (et les textes qui l'utilisent) va biaiser l'analyse purement archéologique, parce que le cadrage et le contexte du cliché jouent un rôle décisif. On commente ainsi le sexe, on invente un âge, on déduit un statut social et une histoire. La description de ces moulages est pleine d'émotion et de subjectivité, à l'image de la phrase introductive de l'ouvrage de T.H. Dyer ${ }^{56}$, grand amateur et utilisateur de photographies dans sa publication sur Pompéi : «And more ghastly and painful, yet deeply interesting and touching objects, it is difficult to conceive. We have death itself moulded and cast the very last struggle and final agony brought before us. They tell their story with a horrible dramatic truth that no sculptor could ever reach ${ }^{57}$. » Dans le rapport de fouilles de Fiorelli, on constate qu'il se livre lui-même parfois à des surinterprétations en recréant l'histoire des morts ${ }^{58}$.

Pour prendre leurs vues, les photographes professionnels replacent notamment les corps hors de leur contexte de découverte et de conservation ${ }^{59}$, sur une table ou au sol, en retournant éventuellement le moulage de façon à le rendre plus expressif et pathétique, ce qui suscite des commentaires apocalyptiques qui s'écartent volontiers de la froide analyse archéologique ${ }^{60}$. La photographie du moulage

55. Archives de 1868 : il envoie une lettre au Ministère de l'Instruction publique, avec une « copie fotografiche » de ses moulages récents. Voir le texte de la lettre de Fiorelli dans Poli Capri, 1996, vol.1, p. 81.

56. Il est un précurseur puisqu'il publie 18 photos mêlées à des gravures dans The ruins of Pompeii: a series of eighteen photographic views, with an account of the destruction of the city, and a description of the most interesting remains, 1867. Mais les moulages n'y figurent pas. Dans son autre ouvrage de 1867, Pompeii: its history, buildings, and antiquities, réédité plusieurs fois dans les années suivantes, il consacre des pages aux moulages, avec une gravure tirée d'une photographie de Sommer, p. 476, en pleine page, commentée p. 477.

57. Dyer, 1870, p. 477.

58. Fiorelli, 1875 , p.452-453, à propos du $8^{e}$ moulage, en 1873.

59. Avant la création d'un petit musée pour abriter les moulages (projet en 1873 , fini en $1875 ; 12$ moulages en 1888), Fiorelli utilise une maison fouillée au XVIII ${ }^{e}$ siècle, appelée "maison des squelettes ou cadavres moulés " à côté de l'École archéologique en VI.17.23-25, fouillée en 1750.Cf. Breton, 1870, p. 272-273. De Carolis, 1998, p. 75-123. Boscoreale Antiquarium exhibition catalogue: The Casts, 2010, p. 7. Dwyer, 2010.

60. Brogi, à cet égard, est une exception, car il prend les corps dans le petit musée de façon surplombante dans les vitrines, ce qui leur ôte leur côté pathétique et montre les faiblesses techniques des moulages - disproportions, déformations - et lacunes ( ${ }^{\circ} 5573$ à 5580). Pour ne prendre qu'un exemple, nous citerons le n 5577, « la femme enceinte », 
$n^{\circ} 4$ (figure 17a), dit « de la femme enceinte », faite par M. Amodio le montre sur une pierre, devant le Vésuve fumant, rappel volontaire de la catastrophe de 79 et de la menace contemporaine ${ }^{61}$. On ne voit pas les vestiges, seuls les débris volcaniques forment un mur derrière la victime.

Très diffusée, cette photo devient même l'illustration de couverture du Guide illustré de Pompéi de Scafati (édition 1876-1877) (figure 17b), devenant un symbole du site et de la catastrophe de 79. Des dessins en sont tirés pour figurer dans les ouvrages publiés sur Pompéi ${ }^{62}$. Sa mise en scène laisse place à des interprétations fantaisistes sur l'histoire potentielle de la femme «enceinte » au moment de la catastrophe $^{63}$ et sur ses derniers gestes car elle a un bras levé et le poing serré : Marc Twain, suite à sa visite en 1867, parle de " mortelle terreur », de « désespoir sauvage ${ }^{64}$ ». M. Monnier écrit en 1864 : « on sent qu'elle s'est débattue longtemps dans d'horribles souffrances : son attitude est celle de l'agonie, non celle de la mort, [...] on voit surtout sa dernière heure comme si on était là, sous la colère du Vésuve ${ }^{65}{ }$. La photographie des moulages semble saisir l'unicité d'un moment précis, celui entre l'agonie et la mort, comme si le photographe s'était trouvé être un témoin de la scène juste pendant l'éruption de 79.

Les quatre premiers moulages réalisés, trouvés dans la même rue, sont traités arbitrairement comme une famille ${ }^{66}$ dont on invente l'histoire avec beaucoup de pathos, à l'exemple d'E. Delaunay qui y voit le mari, la femme et les deux filles : « Le premier cadavre (Vitrine I) est celui de la femme : elle est tombée sur le dos. Bien que ses traits soient peu distincts on reconnaît qu'elle a souffert et qu'elle a été étouffée. Son visage cherche l'air et sa tête semble se soulever vers le ciel. La main droite crispée s'appuie sur la terre le bras gauche veut repous-

qui, vue de dessus et dans une vitrine, s'avère un moulage peu réussi, sans traits du visage, déformé et donc peu évocateur.

61. M. Amodio fait aussi une photographie du moulage $n^{\circ} 1$ devant le Vésuve (Kelsey Museum of Archaeology), mais elle est moins diffusée. G. Sommer fait une vue des moulages 2 et 3 posés sur une table, avec la regio VI derrière.

62. Delaunay, Promenade à Pompéi, 1877, 24 p.

63. Le passage au scanner en 2015 a démontré qu'elle n'est pas enceinte et que le moulage est imparfait, créant une bosse.

64. «The woman had her hands spread wide apart, as if in mortal terror. And I imagined I could still trace upon her shapeless face something of the expression of wild despair that distorted it when the heavens rained fire in these streets so many ages ago. »

65. M. Monnier, Pompéi et les Pompéiens, 1864, 416.

66. Delaunay, 1877; Dyer, 1870.
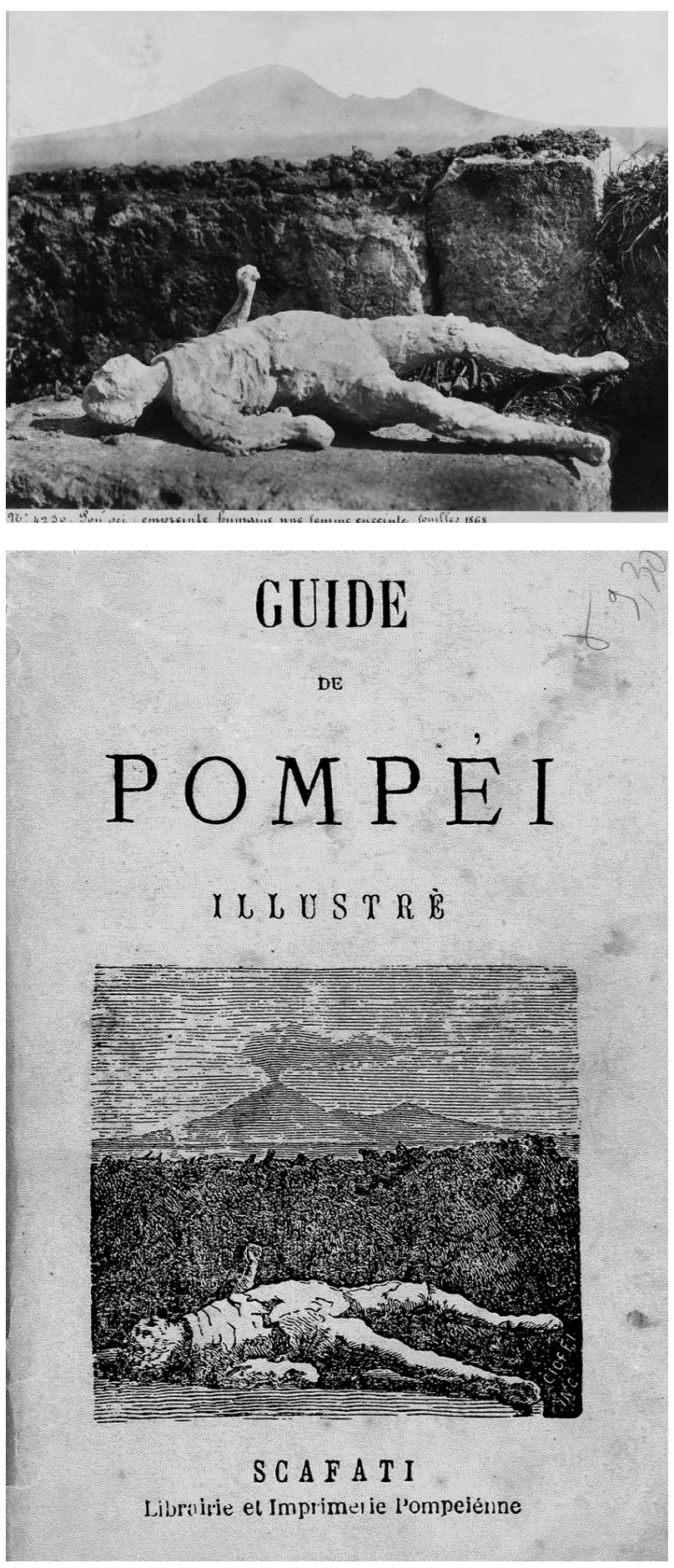

Figure 17 : (a) M. Amodio, n 4230. «Pompéi. Empreinte humaine : une femme enceinte, fouilles 1868 [sic]. " Moulage $n^{\circ} 4$ de février 1863; (b) Couverture du guide Scafati, 1877.

(a) M. Amodio, $n^{\circ}$ 4230. "Pompéi. Empreinte humaine : une femme enceinte, fouilles 1868 [sic]." Cast of february 1863, $n^{\circ} 4$; (b) Scafati guide's cover, 1877. 
ser un ennemi invisible. Cet homme d'un certain âge était, comme je l'ai dit le père des deux jeunes filles qui le suivaient à quelques pas et qui moururent ensemble. Ces deux sœurs couraient en se soutenant l'une l'autre : respirant le même poison elles s'affaissèrent les pieds enlacés et en même temps. (Vitrine V). La plus âgée s'est couchée sur le côté comme pour dormir; l'autre (elle n'avait pas quatorze ans) est tombée sur le ventre en étendant ses bras comme une protection. Une main crispée et dont les ongles s'enfoncent dans la chair, atteste la souffrance; l'autre main tient serré sur le visage un pan de robe ou un mouchoir, comme si la pauvre petite avait espéré se préserver du souffle méphitique ses deux pieds battent l'air, pris dans les plis de la tunique. Ce tableau pathétique est un drame tout entier; c'est un groupe d'un mouvement vrai, d'une expression saisissante, la nature a été moulée sur le vif entre l'agonie et la mort. »

Cela justifie la publication simultanée des victimes 1, 2 et 3 "d'après une photographie de G. Sommer » dans un article de L'Illustration en septembre $1863^{67}$, qui s'intitule "Cadavres retrouvés à Pompéi » (figure 18a-c). Les photos des victimes $n^{\circ} 2$ et 3 (figure 18a), appelés « la femme et sa fille », qui composent un duo poignant, servent d'illustrations suggestives à des scénarios de la catastrophe imaginés par Marc Monnier et E. Delaunay ${ }^{68}$. Or Sommer a réalisé une seconde photographie avec deux hommes qui observent les moulages : le contraste entre le passé et le présent, entre la vie et ce qui évoque la mort est ainsi souligné par cette juxtaposition (figure 19).

La position la plus dramatique (figure 20) est choisie par les photographes pour montrer l'horreur de la catastrophe et de l'agonie ${ }^{69}$. Le moulage d'un homme tombé face contre terre, seul réussi parmi sept squelettes trouvés le 12 mars $1868^{70}$, est photographié non pas dans sa position de découverte archéologique, mais face vers le ciel, de manière à mettre en évidence le visage qui semble terrifié, avec

67. No 1073, 19 septembre 1863, p. 208. Il s'agit d'un montage tiré de deux photographies différentes de G. Sommer.

68. Monnier publie un dessin de Thérond, tiré de la photographie des deux moulages par Sommer. Delaunay, 1877, p. 25.

69. Il est frappant de constater que dans le cas de «l'homme paisible », on n'a pas retourné le corps pour les photos.

70. Le rapport de fouille de Fiorelli indique qu'il est trouvé «face contre terre, dans un geste ultime de tentative de respirer, étouffé par les fumées ", lors de la fouille d'une pièce de la maison de Marcus Gavius Rufus (VII 2, 16). le squelette visible et le rictus saisissant, les dents serrées, les bras levés.

L'éloquence d'Ernest Breton, qui fait reproduire dans son ouvrage de 1868 un dessin tiré de cette photographie, est évocatrice de la dramatisation extrême en usage à l'époque : "Ce moulage, le mieux réussi de tous à l'exception de la jambe gauche qui n'a pu être reproduite, est aussi celui
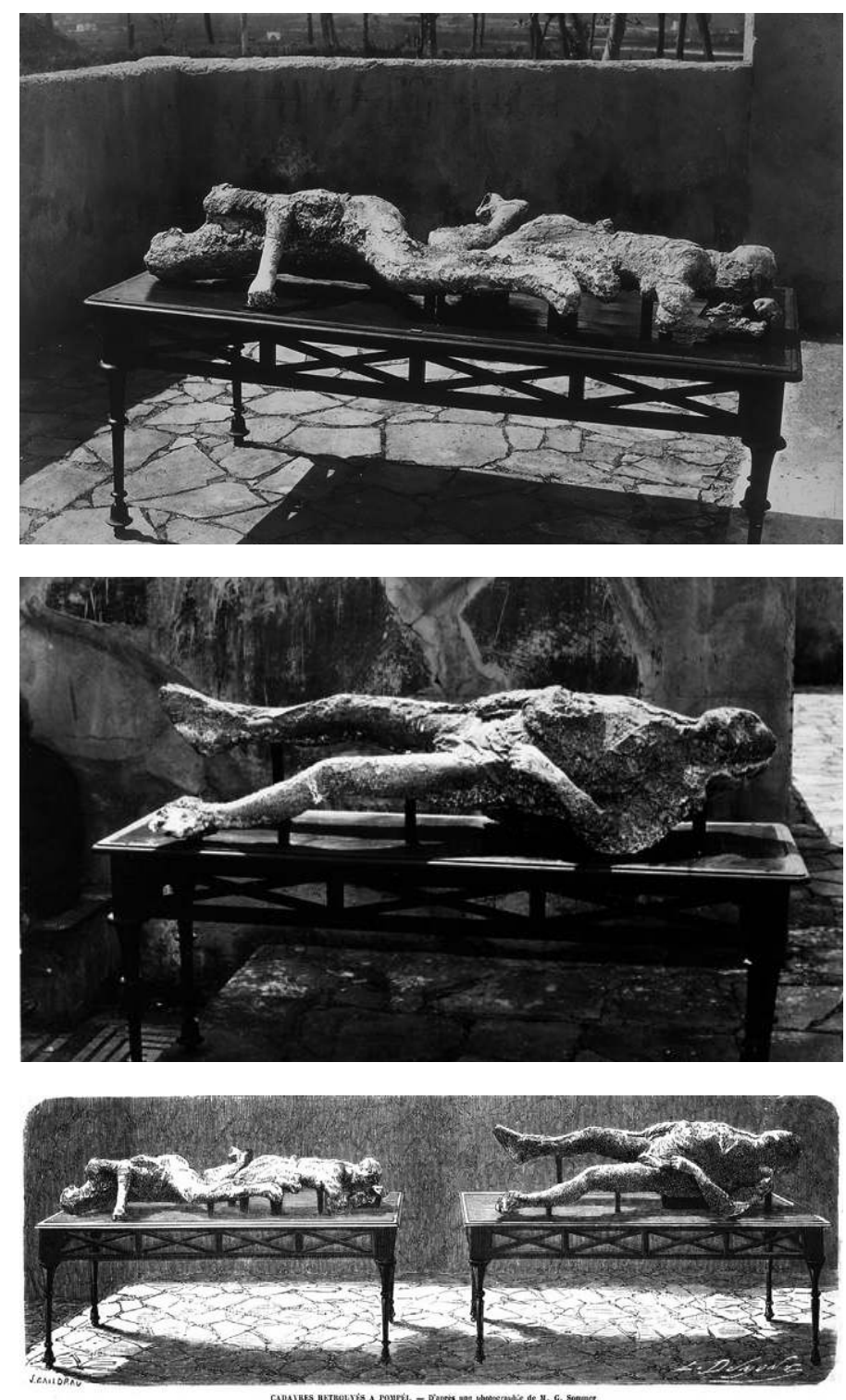

Figure 18 : (a) G. Sommer, $n^{\circ}$ 1242. Moulages $n^{\circ} 2$ et 3 (la femme et sa fille); (b) G. Sommer, $\mathrm{n}^{\circ}$ 1243. Moulage $\mathrm{n}^{\circ} 1$ (l'homme); (c) Moulages $\mathrm{n}^{\circ} 1$, 2 et 3, dans L'Illustration, du 19 septembre 1863, d'après des photographies de Sommer.

(a) G. Sommer $n^{\circ}$ 1242. Casts $n^{\circ} 2$ and 3, (B) (the woman and her daughter); (b) G. Sommer, $n^{\circ} 1243$. Cast $n^{\circ} 1$ (the man); (c) Casts $n^{\circ} 1$, 2, 3 in L'Illustration, 1863, September 19th from Sommer's pictures. 


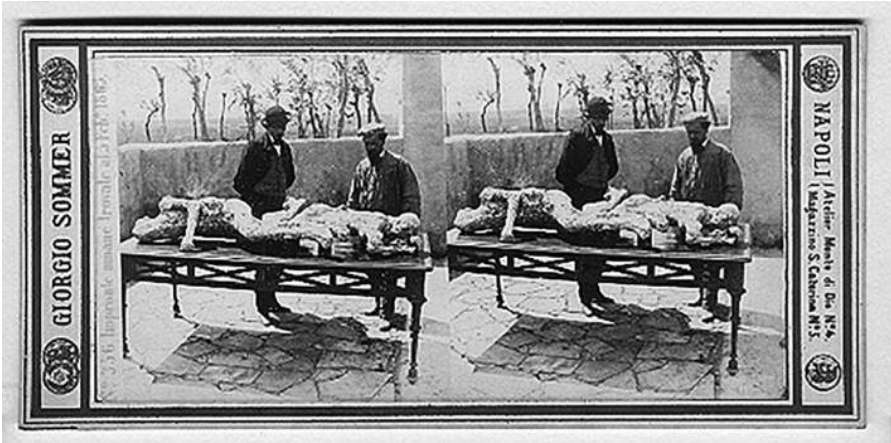

Figure 19 : G. Sommer, nº 336. Photographie stéréoscopique des moulages $\mathrm{n}^{\circ} 2$ et 3 .

G. Sommer, $n^{\circ} 336$. Stereoscopic view of casts $n^{\circ} 2$ and 3 .

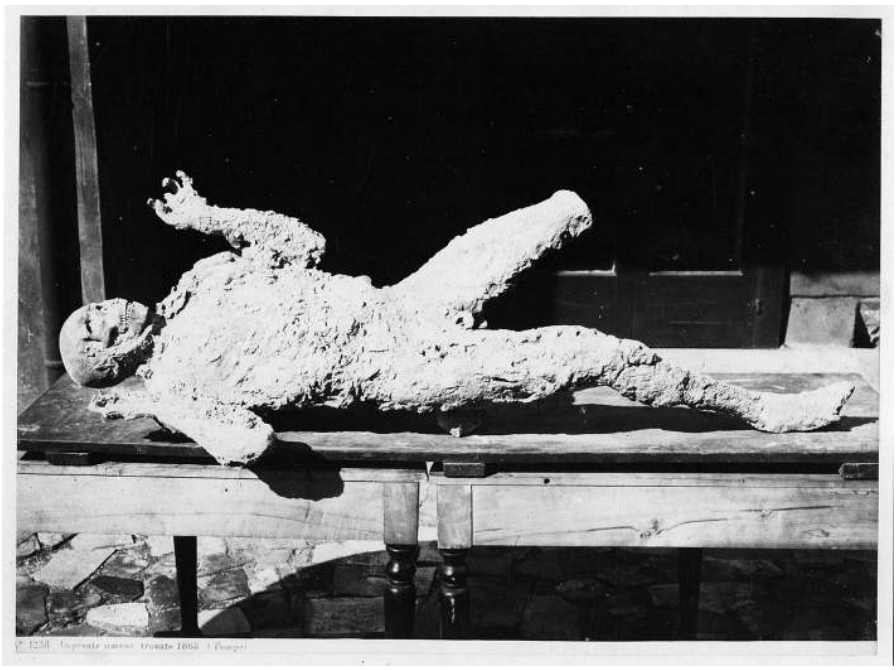

Figure 20 : G. Sommer, $\mathrm{n}^{\circ}$ 1238. Moulage d'un homme en 1868. (=Sommer n 339 en stéréoscopie). Une jambe n’a pas réussi à être moulée. De ce fait, des interprétations fantaisistes fleurissent sur la cause de la mort : on avance que c'est un amputé d'une jambe qui n'a pas pu s'échapper comme d'autres... (Neville-Rolfe, 1888).

G. Sommer, $n^{\circ}$ 1238. Cast of a man, in 1868. One leg failed to be molded. As a result, fanciful interpretations flourish on the cause of death: it is argued that it is a leg amputee who could not escape (Neville-Rolfe, 1888).

dont l'expression est la plus terrible; c'est la personnification du désespoir poussé à son dernier paroxysme $^{71}$. » La surinterprétation des positions des bras contractés et pliés (dite aujourd'hui « attitude du pugiliste »/nociceptive ou flexor reflex ${ }^{72}$ ), notamment à cause des photographies où le corps fut posé à l'envers, fut la cause d'une erreur tenace sur d'ultimes gestes de défense, alors qu'il est prouvé aujourd'hui que la plupart sont morts

71. Breton, 1868

72. Petrone, 2005, p. 36. suite à une violente hyperthermie due à la nuée $\operatorname{ardente}^{73}$

Les photographes replacent aussi par la suite d'autres moulages dans des pseudo-remises en contexte dans la ville, loin de leur véritable position lors de la découverte archéologique ou de la simple présentation en vitrine dans laquelle les visiteurs du site voient les corps. Malgré leur découverte à la Porte de Stabies, c'est dans le quadriportique du théâtre que deux corps ${ }^{74}$ sont mis en scène par les studios professionnels. Sur toutes les vues, on a retourné la femme, les bras levés, pour rendre le couple plus pathétique ${ }^{75}$, alors que lors de la fouille, elle a été trouvée à plat ventre, comme en atteste le journal des fouilles. Les éditions Esposito les montrent au sol, devant un Vésuve toujours menaçant, souligné par la couleur ajoutée à l'aquarelle et le panache accentué, en version colorisée (figure $21 \mathrm{~b}$ - planche couleur IX), alors que sur l'original monochrome, on ne voit même pas le Vésuve (figure 21 a - planche couleur IX). La colorisation donne de l'intensité à la démonstration de la menace apocalyptique du volcan.

Par leur mise en scène des corps moulés in situ, ces photographies dépassent la simple fonction mémorielle, elles mènent à la recherche du pourquoi et du comment de la mort, à la compréhension de la catastrophe et de son vécu. On se met à partager les derniers moments des Pompéiens.

\section{Artefacts, photographie et ouvres d'art}

En 1863, Fiorelli a donc créé une nouvelle catégorie d'objets avec ses moulages. Il est difficile d'établir au niveau émotionnel si on est devant une statue, une personne, une pièce archéologique ou un double (Osanna, 2015, p. 244). Cela trouble, intrigue, séduit.

73. Le choc thermique est d'au moins $200 / 250^{\circ} \mathrm{C}$, ce qui cause une forte contraction musculaire. La comparaison a été faite avec des morts de la montagne Pelée, en Martinique, en 1902 ou du Mont Lamington en Nouvelle Guinée, en 1951 (Lazer, 2017, p. 88). Certains sont morts suite à l'effondrement des charpentes : dans ce cas, la contraction musculaire n'est pas visible. Petrone, 2005, p. 34-37.

74. Ce sont les $14^{\mathrm{e}}$ et $15^{\mathrm{e}}$ corps moulés par Fiorelli et ce sont ceux d'un homme et d'une femme (Garcia y Garcia, 2006, 193-194; Dwyer, 2010, 103-107).

75. Si l'on lit le Guide de Pompéi par Fiorelli lui-même (1897), p. 92-93, la description est très technique, et excepté « les mains crispées » de l'homme (inv. 43), il n'y a aucun pathos : il décrit l'aspect du corps, sa position, son vêtement, le site de découverte. 
Planche IX (Delphine ACOLAT - Mémoire de la catastrophe volcanique de Pompéi...)
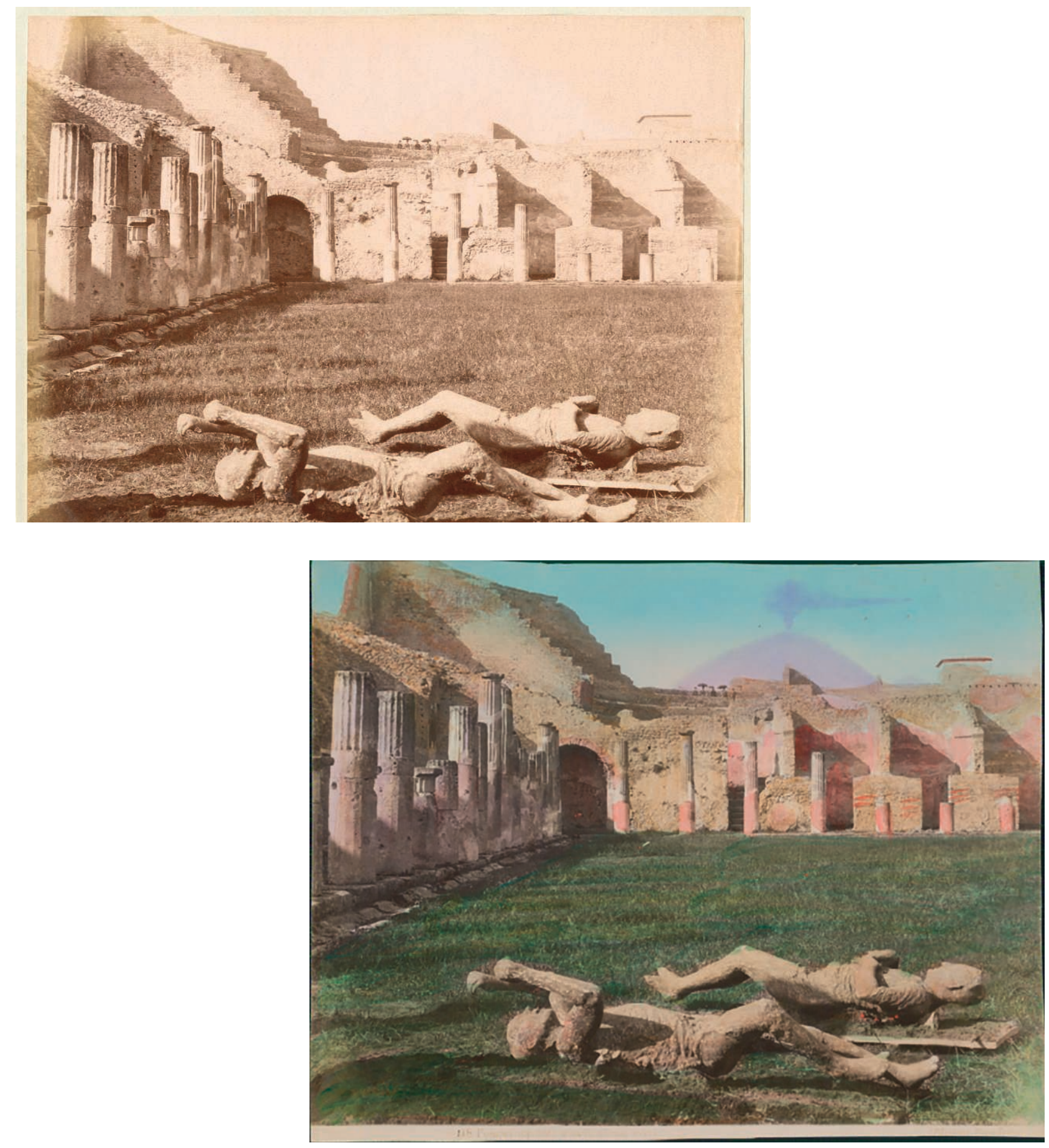

Figure 21 : Éditions Esposito. Moulages n ${ }^{\circ} 14$ et 15 dans le quadriportique du théâtre. (a) version monochrome où on ne distingue pas bien le Vésuve; (b) version colorisée avec le Vésuve accentué symboliquement.

Esposito editions. Casts $n^{\circ} 14$ and 15, in the quadriporticus of the theater. (a) black and white picture; (b) Colorized picture with pronounced Vesuvius. 

En 1870, Thomas Dyer écrit à propos du moulage de jeune fille $\left(n^{\circ} 4\right)$ : «Her legs are drawn up convulsively; her little hands are clenched in agony... The form of her head is perfectly preserved. The texture of her coarse linen garments may be traced, and even the fashion of her dress, with its long sleeves reaching to her wrists; here and there it is torn, and the smooth young skin appears in the plaster like polished marble [...] The cast would seem to be taken from an exquisite work of Greek art ${ }^{76}$. » La démarche des photographes permet de poser en effet la question de la façon dont on considère les moulages : artefacts? Reliques? Euvres d'art? En plus de la réinvention d'une histoire, certaines mises en scène photographiques des moulages blancs en plan serré devant un drap noir évoquant le deuil concourent à en faire également des œuvres d'art ${ }^{77}$ qui sont confrontées à l'idéal du classicisme, à cause de leurs formes tourmentées, de leur imperfection, du plâtre au lieu du bronze ou du marbre. Comme l'a souligné J. Seydl (2012), la forte diffusion ${ }^{78}$ via la photographie de ces «modèles » nouveaux joue un rôle dans la révolte, fin $\mathrm{XIX}^{\mathrm{e}}$ siècle, contre le langage conventionnel de la sculpture classique. Ces corps "sculptés » sont nés d'un vide et non d'un artiste, et leur apparition peuple désormais Pompéi d'une mort palpable, de présences fantomatiques. Le plus frappant est certainement le moulage du chien de la maison de Vesonius Primus ${ }^{79}$, resté célèbre depuis le 20 novembre 1874 et très reproduit après la photographie de Sommer (figure 22).

Les photographies des moulages ont d'ailleurs donné lieu à la conception d'œuvres d'art contemporain beaucoup plus récemment ${ }^{80}$. Robert Rauschenberg, en 1956, inclut la photographie du chien par Sommer dans sa composition Small rebus ${ }^{81}$ qui travaille le rapport au temps et à la mémoire dans l'histoire de l'art. Allan McCollum crée The Dog from Pompeii en 1991. Il a multiplié

76. Dyer, 1870 , p. 478

77. Sommer $n^{\circ}$ 1282. « Pompei. Inpronte humane». Voir Hales 201 1, p. 160.

78. Nous avons examiné un certain nombre d'albums sur Naples et Pompéi : à chaque fois, plusieurs moulages y figurent.

79. Appelée aussi maison d'Orphée, Regio VI, Insula 14, n. 20.

80. Allan McCollum, The Dog from Pompeii, 1991. Polymère. $53 \times 53 \times$ $53 \mathrm{~cm}$. Friedrich Petzel Gallery, New York. Antony Gormley, Untitled, 2002. Mild steel blocks, $45 \times 189 \times 53 \mathrm{~cm}$. Collection of Asher Waldfogel and Helyn MacLean.

81. Small Rebus, 1956. Peinture à l'huile, graphite, échantillons de tissu peints, papier, journal, coupures de magazines, photographies en noir et blanc, fragments de carte des Etats-Unis,tissu et timbres sur toile, $88,09 \times 116,8 \times 4,4 \mathrm{~cm}$. Museum of Contemporary Art, Los Angeles, The Panza Collection.

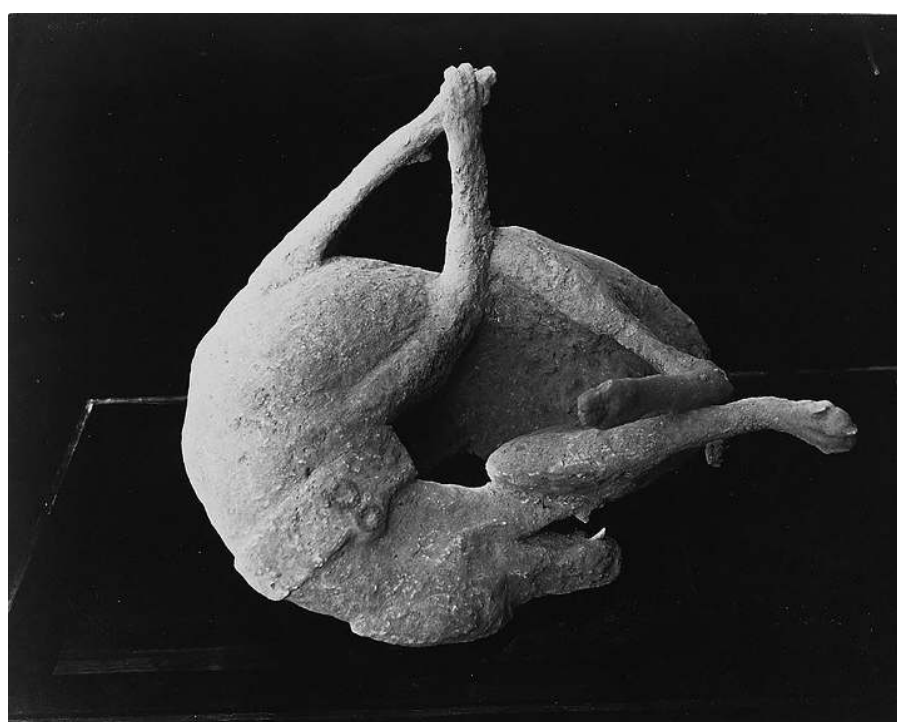

Figure 22 : G. Sommer, $n^{\circ} 1287$ ( $n^{\circ}$ 5372). Moulage du chien de Pompéi. $19,5 \times 25,2 \mathrm{~cm}$, tirage albuminé. Le passage au scanner a révélé une surprise de taille pour ce moulage : aucun élément de squelette ne se trouve à l’intérieur (Lazer, 2017, p. 6).

G. Sommer, $n^{\circ} 1287$ ( $\left.n^{\circ} 5372\right)$. Cast of the dog of Pompeii. The $x$ scanner revealed a major surprise for this cast: no skeletal element is inside (Lazer, 2017, p. 6).

par dizaines le chien d'après un moule fait sur l'original. Ses chiens sont blancs et présentés sur fond noir, comme sur les photographies anciennes, mais avec des angles de présentation qui diffèrent pour des chiens identiques. Antony Gormley a copié les moulages humains en 2002 à partir de petits blocs d'acier assemblés. Il déclare en 2010 que les moulages originaux sont une modélisation de la fin de l'humanité et lui ont évoqué la fin du monde : «The bodies each lie or crouch in their unique position of facing death, but they do not have the morbidity of the mummy, more a direct existential appeal reminding us that life can end at any time, whether during a trip to the shops or while sleeping [...] The abstracted bodies of Pompeii A.D. 79 model the end of the human project and give us a foretaste of our own demise, in the manner of the dinosaur skeleton but much more immediate. I made three pieces, inspired by a visit in 2002, all of which try to think about a lack of air, fallout, and the end of time. »

Ainsi, la confrontation entre le volcan et les moulages crée un nouveau rapport entre le site archéologique et la photographie, qui se pose en témoin privilégié et sensationnel de l'agonie des habitants, et conforte l'image de paradigme de l'apocalypse de Pompéi en y ajoutant le frisson du sublime. Si 
les progrès de la science archéologique sont servis, dans leurs répercussions médiatiques, par la photographie, le succès des clichés des moulages prouve que l'éruption de 79 reste bien l'ultime modèle éruptif : la cité est désormais montrée comme revivant constamment l'éruption passée, et sous une menace constante. Le Vésuve représente la catastrophe du passé, du présent et de l'avenir.

\section{Conclusion}

Ce que les voyageurs voient en montant sur le Vésuve et que la photographie relaie, c'est un spectacle attrayant, qui représente la vie de la Nature et sa force, face à la petitesse de l'homme. Les photographes ont donné une fonction informative à leurs vues, montrant aussi la force destructrice des éruptions. À travers leurs fonds, on constate que la mort est d'abord invisible, puisqu'ils excluent volontiers l'humain du site de Pompéi sur leurs clichés. Elle devient ensuite palpable, saisissante, avec un travail sur le cadrage et la mise en scène des moulages des morts, qui va être repris comme une référence dans les publications et va accroître encore la fascination pour le site, en y ajoutant du morbide. Le panache du Vésuve fumant et l'absence d'êtres vivants sont donc un rappel volontaire, par les photographes, de ce risque de catastrophe omniprésent dans la baie de Naples, et font de Pompéi un paradigme de l'apocalypse et une cité peuplée de fantômes ${ }^{82}$.

L'instantanéité du media reproductible qu'est la photographie sert à démontrer l'actualité du risque et du danger de la nature de ce volcan, toujours actif, qu'elle décrit dans l'action et dans le détail, tout en se faisant l'écho de la mort des Pompéiens qu'elle rend sensationnelle, pathétique et objet de questionnements sans cesse renouvelés ${ }^{83}$.

Loin du site, dans les années 1870, James Pain recrée, à Londres puis en tournée aux Etats-Unis

82. Evoqué dans la presse pour San Francisco en 1906, pour le 11 sept 2001 (dans le Wall Street Journal), et pour Fukushima en 2011.

83. En 1903, dans sa publication sur l'éruption de la montagne Pelée (île de la Martinique) de 1902, Alfred Lacroix, illustrera très largement son propos de photographies inédites prises par lui-même, sa femme et d'autres photographes avant, pendant et après l'éruption. En introduction, il écrit (p. 7) : «Toutes ces photographies étant données comme documents, il m’a paru nécessaire de sacrifier le côté artistique à l'absolue sincérité et de les reproduire sans retouches. » Dans sa planche XIX, il présente la ville de Saint Pierre dévastée par la nuée ardente de mai 1902, dont seuls quelques murs tiennent encore debout. Les deux vues, depuis le sud et l'ouest, sont très comparables aux panoramas de Pompéi, avec les rues totalement vides, l'absence de toit, les murs éventrés. La photographie joue un rôle capital dans ce premier rapport de vulcanologie.
(Gardner Coates, 2012) ${ }^{84}$, la destruction de Pompéi (The Last days of Pompeii), avec un spectacle pyrotechnique (pyrodrama) qui se veut sans concurrence ( « The ancient city will be destroyed again tonight gorgeaous scenery. The greatest, most sublime spectacle on earth. The whirlwind of death mingled with floods and flame », proclament les affiches $\left.{ }^{85}\right)$. La violence «sublime » de la nature et la mort seront désormais les objets d'un travail esthétique dont les photographes ont été les précurseurs.

\section{Annexe : Guide Baedeker}

\section{Guide Baedeker (édition de 1896) sur l'ascension du Vésuve}

"De Resina, il y a une bonne route qui se détache à g. de la route de Salerne, au-delà de l'entrée des fouilles d'Herculanum. Les vignes luxuriantes où l'on passe produisent le fameux vin muscat connu sous le nom de "lacryma-christi". Les paysans vous en offrent à leurs portes; on fera bien de n'en accepter qu'au retour (1 fr.). Il faut payer en petite monnaie, parce que ces gens refusent ordinairement de rendre sur de grosses pièces. Plus haut, quand le regard n'est plus arrêté par des murs de jardins, on découvre une vue splendide. Au bout d'env. 3/4 d'h., on atteint l'énorme coulée de lave foncée de 1872, que l'œil suit jusqu'à S. Sebastiano et Massa di Somma. Le chemin, qui s'élève en faisant de grandes courbes, le traverse plusieurs fois.

A 1 h 1/2 de Resina, on est à l'ermitage et à l'observatoire météorologique, sur la croupe qui divise en deux bras la coulée de lave descendant du Vésuve, à $676 \mathrm{~m}$. d'altitude. Cet observatoire renferme les instruments ordinaires et un appareil spécial pour l'observation des tremblements de terre, le séismographe. Il a été d'abord dirigé par Melloni, puis par Palmieri. Une plaque à l'entrée du bâtiment rappelle le souvenir des personnes qui ont alors péri dans l'Atrio del Cavallo en 1872. Palmieri est alors resté intrépidement à l'observatoire. Il y a près de là une auberge convenable. La route publique se termine à 5 min. de l'observatoire. Le reste, jusqu'au pied du cône de cendres (env. 3 kil.), a été fait en 1879-1880 par la compagnie du chemin de fer funiculaire, et l'agence Cook, qui l'a acquise en 1889 avec le funiculaire, n'y

\footnotetext{
84. Le spectacle passe aux États-Unis à partir de 1879. Il est joué à New York (Coney Island) jusqu'en 1914

85. The Minneapolis Tribune, 28 août 1890, p. 6. 
laisse passer, avec des voitures autres que les siennes, à cheval ou à pied, que moyennant 5 fr. par personne. Une partie de la route ayant été détruite, en 1895, par les laves à une distance de 300 mètres, l'agence Cook $y$ fait faire le trajet à cheval sans hausser les prix. La route se termine à la station inférieure du funiculaire, à env. 800 m. d'altitude (buffet; dé. 4 fr., di. 6, v. c.). Un employé vous délivre à l'arrivée des billets pour le chemin de fer. Mais on n'est pas obligé de partir immédiatement, s'il doit y avoir de la place dans le train suivant. [...] Les piétons et les cavaliers venus de Resina doivent, s'ils veulent éviter les frais considérables de péage et de chemin de fer, en gravissant tout le cône, quitter la route au bureau de Cook et prendre l'ancien chemin raboteux, par lequel on arrive en 3/4 d'heure dans le voisinage de la station inférieure. Là on traverse gratuitement la route et l'on commence à monter immédiatement au sud de la gare. Les cavaliers quittent leurs montures au pied du cône de cendres. L'ascension est très pénible et demande 1 h. à 1 h. 1/4. [...] Au retour, on descend très rapidement, en sautant sur la cendre, en 10 min. au pied du cône. »

\section{Bibliographie}

Breton E., 1868. Pompeia décrite et dessinée par Ernest Breton [...] suivie d'une Notice sur Herculanum, Paris.

Breton E., 1870. Pompeia, Guide de visite à Pompei, $3^{\text {rd }}$ ed. Paris.

Chevalier C., 1871. Naples, le Vésuve et Pompéi, croquis de voyage, Tours.

Cocco S., 2007. Natural Marvels and Ancient Ruins: Volcanism and the Recovery of Antiquity in Early Modern Napels, in Gardner Coates V. C., Seydl J.L. (éds.), Antiquity recovered. The Legacy of Pompeii and Herculaneum, Los Angeles, J. Paul Getty Museum, p. 15-35.

Cocco S., 2013. Watching Vesuvius: A History of Science and Culture in Early Modern Italy, Chicago-London.

De Carolis E., Patricelli G., Ciarallo A., 1998. Rinvenimenti di corpi umani nell'area urbana di Pompei, Rivista di Studi Pompeiani, vol. 9 (1998), p. 75-123.

De Keratry E., 1867. Les ruines de Pompéi, Paris.

Delaunay E., 1877. Promenade à Pompéi, Scafati.

Du Pays A.J., 1853, rééd. 1855, 1859, 1865. Guide de l'Italie, Paris.

Dwyer E., 2007. Science or Morbid Curiosity? The Casts of Giuseppe Fiorelli and the Last Days of Romantic Pompeii, in Gardner Coates V., Seydl J. L. (eds.), Antiquity Recovered: The Legacy of Pompeii and Herculaneum, Los Angeles, J. Paul Getty Museum.

Dwyer E., 2010. Pompeii's Living Statues. Ancient Roman lives stolen from death, Ann Arbor, University of Michigan Press.
Dyer T.H., 1870. Pompeii: Its History, Buildings and Antiquities, N.Y. Scribner, Welford.

Fanelli G., Mazza B., 2013. Italie, Le Grand Tour. Dans le miroir de la photographie au XIX siècle, Paris.

Fiorelli G., 1875. Descrizione di Pompei, Napoli.

Garcia y Garcia L., 2006. Danni di guerra a Pompei : una dolorosa vicenda quasi dimenticata, Roma.

Gardner Coates V.C., Lapatin K.D.S., Seydl J.L., 2012. The Last days of Pompeii: decadence, apocalypse, resurrection, on view at the J. Paul Getty Museum at the Getty Villa in Malibu, from September 12, 2012, to January 7, 2013, Los Angeles-Cleveland.

Guiraud L., 1872. L'éruption du Vésuve en avril 1872, Recueil de la Société des Sciences, Belles-Lettres et Arts de Tarnet-Garonne, Montauban.

Hales S., 2011. Cities of the dead, in Pompeii in the public Imagination from its rediscovery to today, Oxford, p. 153-170.

Hannary J., 2008. Encyclopedia of nineteenth-century photography, New York/London.

Istituto NAZionale di GEOFisica e vUlCANOLOGia, 2004. Terra viva: Vulcani e terremoti nelle fotografie delle collezioni alinari, Firenze.

Lacroix A., 1903. La montagne Pelée et ses éruptions, Paris.

Layard A.H., 1864. London Quarterly Review (American edition), 115, January-April, p. 161-180.

Lazer E., 2017. "What's in the casts? New horizons and many recent surprises in Pompeian archaeology ", in School of Philosophical and Historical Inquiry Magazine, University of Sydney. [https://adobeindd. com/view/publications/04fa6738-7e41-4719-8904c 833 a826e4f2/7pl5/publication-web-resources/pdf/ Magazine_issue_4.pdf].

Morin J., 2005. Évolution géomorphologique du Vésuve depuis l'époque moderne : apports de l'iconographie et de la cartographie anciennes, mémoire de Master 1 de géographie physique, université de Paris I.

Osanna M., Caracciolo M. T., Gallo L., 2015. Pompéi et l'Europe, Milano.

Pagano N., 1877. Guida di Pompéi, Napoli.

Petrone L.P., 2005. Le Vittime dell'Eruzione del 79 AD, in D’Ambrosio A., Guzzo P.G. (dir.). Storie da un'Eruzione: Pompei, Ercolano, Oplontis: Guida alla Mostra, ed. d'Ambrosio, Milan, p. 33-44.

Petrone P., 2007. Le Vittime ercolanesi dell'eruzione pliniana del 79 A.D., in Itinere. Ricerche di archeologia in Campania. [https://www.researchgate.net/publication/301646908_Le_ vittime_ercolanesi_dell'eruzione_pliniana_del_79_AD].

Poli Capri P., 1996. Pompei, letters and documents, vol.1. Oct. 31, 1863-May 26, 1869, Roma.

Pucci G., 2015. Le plâtre et son écho. Histoire et histoires des calques de Pompéi, in Osanna M. (dir.), Pompéi et l'Europe, 2015. Pompéi et l'Europe, Milano, p. 239-244.

SSANP, 2010. Boscoreale Antiquarium exhibition catalogue: The Casts, 5 March-20 December 2010.

Zanchetti G., 2015. La conformation et la forme, in Osanna M. (dir.), Pompéi et l'Europe, Milano, p. 365-369. 